


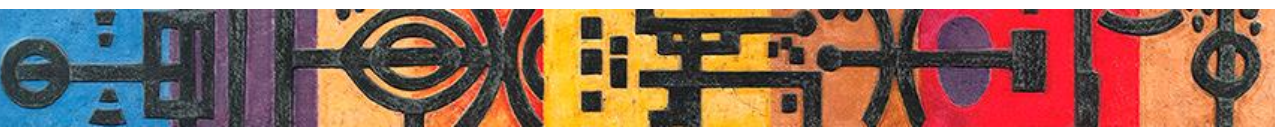

\title{
Zur Translatologie der von Dichtern übersetzten Gedichte: „die grausamsten aller Huldigungen" an das Original?
}

\author{
Larisa CERCEL \\ Universität Leipzig
}

\begin{abstract}
The essay addresses George Steiner's reference to Paul Celan's translation of Jules Supervielle's poem Chanson in the context of his account of transfigurative translations that betray the original even as the original is elevated in poetic richness - Celan's translation is cited as a prime example of a translation that compensates for the weaknesses of the original. In this regard, my aim is to question two matters in particular: (1) The apodictic and strongly polarizing style of Steiner's commentary which declares Celan's translation to be the "cruelest homage" to Supervielle's rather "conventional" original; (2) Steiner's conception of the relationship between source and target text as an exercise in aiming at reciprocity and compensation. These two matters will be discussed against the backdrop of contemporary translatological conceptions of the relationship between source and target text. It will be suggested that the critical discussion of Steiner's position heavily depends upon the category of identity: translation — and the original- possess their own literariness and convey a specific poetic statement, and in this case, the objective of this essay is to argue that this should be recognized or acknowledged as true, and that it is not necessary to play the one off against the other.
\end{abstract}

Key words: Hermeneutic Motion, Identity, Poetic Interaction, Paul Celan, Jules Supervielle. 


\section{Einleitung}

Im Mittelpunkt des hermeneutisch orientierten Übersetzungsmodells von George Steiner steht das Verhältnis von Übersetzung und Original, das durch einen vierphasig ablaufenden, komplexen Prozess (ausführlich beschrieben in Nach Babel, Steiner/Plessner 2004: 311-389) in die Herstellung des Ausgleichs und der Reziprozität zwischen den zwei Texten zu münden habe. Dass eine Übersetzung dieser Zielsetzung vollkommen genügt, sei ein äußerst seltenes Phänomen, ja, „ein Wunder des menschlichen Geistes” (ebd.: 389). Die Beispiele, mit denen Steiner die Dialektik der „,beiderseitigen Steigerung“ zwischen Original und Translat (ebd.: 380) veranschaulicht, bleiben alle ebenfalls hinter dem formulierten Ausgleichsdesiderat zurück: Sie „,betrügen” das Original (ebd.: 383) nach unten oder nach oben, indem sie es schmälern oder verklären.

Der vorliegende Aufsatz nimmt das Phänomen der transfigurierenden, nach oben verratenden Übersetzungen von DichterÜbersetzern, das Steiner mit Paul Celans Übersetzung des Gedichts Chanson von Jules Supervielle illustriert, näher in den Blick. „Über Supervielle weit hinausgreifend” (Steiner/Plessner 2004: 387) lege Celan - ,wie es wohl nur bei seinem Genie möglich war” (ebd.: 386) - eine deutsche Version des Gedichts vor, nach dessen Lektüre man „kaum zu Supervielle zurück” (ebd.: 387) könne: Das Translat mache das Original größer und gewichtiger, als es ist - ein Umstand, aus dem George Steiner die Schlussfolgerung ableitet: „Übersetzungen solchen Ranges sind die grausamsten aller Huldigungen" (ebd.: 387).

Der Aufsatz setzt sich mit diesem Kommentar und seinem Duktus im Kontext des gegenwärtigen translatologischen Diskurses über die Relation zwischen Ausgangs- und Zieltext auseinander. Vor dem Hintergrund des Zeugnisses Steiners für die unbestreitbar hohe Qualität der Lyrik und Übersetzungskunst Paul Celans wird eine translatologische Analyse des Gedichts Chanson im Original und Translat vorgenommen, um sowohl die ,eigene Identität” des jeweiligen Textes (Gil 2015: 146) als auch die Modalität ihrer ,poetischen Interaktion” miteinander (Sanmann 2013) näher zu be- 
leuchten. Versucht wird dabei, sachlichere Wege der Deskription der poetischen Leistung und Intention von Ausgangs- und Zieltext sowie ihres Verhältnisses zueinander als die oxymoronische Formel Steiners aufzuzeigen, die als Untertitel der vorliegenden Studie fungiert. Die Analyse weicht in ihrer Schlussfolgerung von der Steiners $a b$.

\section{George Steiner als Leser Paul Celans}

Steiners Hochschätzung für Paul Celan ist bekannt. Schon 1975, dem Jahr der Veröffentlichung der Monographie After Babel, preist er Celan als den ,wohl größte[n] europäische[n] Lyriker nach 1945” (Steiner/Plessner 2004: 195). Kurz darauf bezeichnet er ihn im Essay On Difficulty als einen Dichter „writing poetry comparable in stature to Hölderlin's and Rilke's" (Steiner 1978: 44). Jene Unsicherheit - oder bloß rhetorische Geste? -, die in der Vermutungspartikel (,,wohl der größte") mitzuhören, jene deskriptive Distanz, die im adverbialen Vergleich (,comparable to") mitzulesen ist, weichen 2011, dem Publikationsjahr von Steiners Abhandlung The Poetry of Thought: From Hellenism to Celan, der stark affirmativen Gewissheit: „Celans Beiträge zur deutschen Dichtung und Prosa haben denselben Rang wie jene Hölderlins. Ihre Innovationen gehen über Rilke hinaus" (Steiner/Bornhorn 2011a: 281). Derselbe Band, der das gegenseitige Durchdringen von Philosophie und Literatur zum Thema hat, schließt mit der Begegnung (bzw. der faktisch-menschlichen Vergegnung) Heidegger-Celan am 24. Juli 1967 an der Universität Freiburg, bei einer der letzten öffentlichen Lesungen Celans, sowie mit dem darauf folgenden Besuch Celans auf Heideggers berühmter Hütte in Todnauberg ab. In jenem Treffen, in dem ,souveränes philosophisches Denken” und „souveräne Dichtung " in den Personen Heidegger und Celan "Seite an Seite” gestanden haben (Steiner/Bornhorn 2011a: 286), erblickt Steiner einen „Höhepunkt” der jahrtausendealten Geschichte, ja „das Wesen der Bezüge zwischen Dichtung und Philosophie, zwischen dem Denken und dessen Poetik, denen von den Vorsokratikern und von Platon 
zum ersten Mal Unsterblichkeit verliehen wurde" (Steiner/Bornhorn 2011a: 282).

Bekannt ist ebenfalls Steiners großes Engagement, mit dem er Celans Werk förderte, begleitete und somit gewissermaßen dessen Rezeption im englischsprachigen Raum prägte (vgl. Hallberg 2006). Bereits die erste, aleatorische Begegnung Steiners mit Celans Dichtung in den 1960er Jahren beim Herumstöbern in einer Frankfurter Buchhandlung zwischen zwei Zügen sei eine elektrisierende gewesen: „Ein Band mit Gedichten, dünner als dünn, mit einem Umschlag in strengem Weiß, fiel mir ins Auge. Die allererste Zeile, die ich las, erzählte von einer Sprache ,nördlich der Zukunft'. Diese Zeile durchquerte mich gleich einer stillen Flamme“ (Steiner/ Bornhorn 2011b: 226). Diese tiefe innere Betroffenheit gibt Steiner in seiner charakteristischen intensiven Rhetorik, die durch seine Autorität als Literaturwissenschaftler und -kritiker noch einmal potenziert wird, an seine Leser weiter. Anlässlich der Publikation der englischen Übersetzung der Poeme Celans durch Michael Hamburger (Poems of Paul Celan, Persea Books, 1988) drückt er in The New Yorker (Steiner 1989) eine ungewohnt eindringliche Empfehlung für Celans Dichtung aus einem ,selbstverständlichen Impuls“ aus:

Gehen Sie und kaufen Sie dieses Buch. Bestehen Sie darauf, dass Ihr Buchhändler es bestellt, wenn er es nicht schon getan hat. Borgen Sie es sich von einem Freund aus, solange Sie auf Ihr Exemplar warten. Stehlen Sie es, wenn nötig. Aber gewähren Sie Celan jetzt, da er bis zu einem gewissen Grad im Englischen verfügbar ist, Eintritt in Ihr Leben. Auf eigenes Risiko, im Bewusstsein, dass er es verändern wird. Im Wissen, dass seine Gedichte - wie der rarchaische Torso Apolls in Rilkes berühmtem Poem - die Forderung aussprechen: ^Ändere dein Leben jetzt. Und damit sollte diese Besprechung, und jegliche andere, enden. (Steiner/Bornhorn 2011b: 227).

Im selben Essay attestiert Steiner Celan jene zutiefst transformierende Kraft, die großen, bahnbrechenden Kunstformen wie abstrakter Malerei, Zwölfton- oder atonaler Musik vorbehalten ist: Celans Dichtung werde ,nach und nach unsere Wahrnehmung, unsere Einfühlung erziehen” (Steiner/Bornhorn 2011b: 238). 


\section{Steiners Kommentar zu Supervielles und Celans Chanson}

Der eingangs zitierten Passage aus After Babel, in welcher Steiner sich mit Celans Supervielle-Übertragung auseinandersetzt (Steiner/ Plessner 2004: 386f.), kommt gleichermaßen Gewicht und Tragweite zu: Im Jahr 1975 machte Steiner wohl als einer der ersten auf die Übersetzungstätigkeit Paul Celans aufmerksam und legte somit den symbolischen Grundstein für ein neues literarisches Forschungsfeld. Das Übersetzungswerk Celans entwickelte sich bekanntlich in den Nachfolgejahrzehnten zu einem essentiellen Forschungsschwerpunkt der Literaturwissenschaft, wo es mittlerweile geradezu monographisch behandelt wird (vgl. u. a. Olschner 1985, Lehmann/Ivanović 1997, May 2004, Harbusch 2005, Pennone 2007). In der Übersetzungswissenschaft besteht für Steiners Impuls hingegen noch immer Nachholbedarf: Einige wenige Translatologen nehmen die Übersetzung der Dichtung Celans in andere Sprachen, dabei vorzüglich ins Englische in Augenschein (vgl. Beals 2014, Boase-Beier 2014, 2017); recht selten wird allerdings Celans eigene Übersetzungstätigkeit aus translatologischer Warte thematisiert (vgl. Paepcke 1987; Nouss 2010; Sanmann 2013: $167-$ 239; Cercel 2020, 2021). Der Umstand mag umso mehr verwundern, als das Phänomen der Dichter-Übersetzer, das Paul Celan zweifelsohne prominent illustriert, gegenwärtig zu den hot topics der übersetzungswissenschaftlichen Forschung gehört (vgl. u. a. Lombez 2016, Woodsworth 2017), ohne dass Celans Übersetzungswerk darin als Untersuchungsgegenstand fungiere. Schauen wir uns nun Steiners Kommentar zu Celans Supervielle-Übersetzung näher an, von dem auch für die Translation Studies weiterführende Forschungsanregungen ausgehen können.

Chanson
(Jules Supervielle)
Jésus, tu sais chaque feuille
Qui verdira la forêt,
Les racines qui recueillent
Et dévorent leur secret,

Chanson

V. 1 Jésus, tu sais chaque feuille

V.2 Qui verdira la forêt,

V.3 Les racines qui recueillent

V. 4 Et dévorent leur secret,

\section{Chanson \\ (Paul Celan)}

Jesus, du kennst sie alle: das Blatt, das Waldgrün bringt, die Wurzel, die ihr Tiefstes aufsammelt und vertrinkt, 
V. 5 La terreur de l'éphémère

V. 6 À l'approche de la nuit,

V.7 Et le soupir de la Terre

V. 8 Dans le silence infini.

V. 9 Tu peux suivre les poissons

V.10 Tourmentant les profondeurs,

V.11 Quand ils tournent et retournent

V.12 Et si s'arrête leur cœur.

V.13 Tu fais naître des chansons

V.14 Si loin au delà des mers

V.15 Que la fille qui les chante

V.16 En tremble au fond de sa chair.

V.17 Écoutez-le bien, demain,

V.18 Jésus aura oublié,

V.19 Ne sera qu'une statue

V. 20 Peinte sur la cheminée. die Angst des Taggeschöpfes, wenn es sich nachthin neigt, das Seufzen dieser Erde im Raum, der sie umschweigt. Du kannst den Fisch begleiten, dich wühlen abgrundwärts und mit ihm schwimmen, unten, und länger als sein Herz.

Du weckst das Lied von jenseits der Meere, dass es lebt, und die's nun singt, muss singen, durchzittert und durchbebt.

Hör gut zu morgen, denn er, er selber weiß es nicht mehr. Ein Holzbild, so seht ihr ihn dastehn auf dem Kamin.

George Steiners Anmerkungen zu Celans bzw. Supervielles Chanson sind knapp und bereits zu Beginn kategorisch wertend: Jules Supervielle selbst schätzt er als „,eine einprägsame, aber nicht erstrangige literarische Figur" (Steiner/Plessner 2004: 386) ein. Sein Gedicht Chanson sei ,wohlgestaltet, aber nicht frei von Banalitäten“ und „Stereotypen“, während Celan „,verdichtet und steigert, wie es wohl nur bei seinem Genie möglich war” (ebd.). Belegt sieht Steiner seine These an diesen Stellen im deutschen Text (alle folgenden $\mathrm{Zi}$ tate stammen aus Steiner/Plessner 2004: 386-387):

- V. 2/V. 3: Durch die Singulare „Blatt“ und „Wurzel“ werde die Anrufung ,erschütternd unmittelbar“.

- V. 3: „Ihr Tiefstes“ sei anders als ,secret“ sowohl abstrakt als auch bildlich und dadurch „doppelt genau“.

- V. 4: „[V]ertrinken“ verstärke die doppelte Präzision, während „dévorer“ „zufällig oder auch nur sonor“" wirke;

- V. 5-8: „[L]'éphémère“ sei ,schweifend unheimlich“ und die darauf folgenden Verse „konventionell“, während Celan hier eigentümliche „Engführungen“ wie „Taggeschöpf“, „nachthin“, ,umschweigen“ bringe, die ,vor ihm nicht da gewesen sind“. Während das ,infini““ im Original ,abgeschwächt“ wir- 
ke, erfülle die Übersetzung - ,über Supervielle weit hinausgreifend” - die „Intention von Schwere und Verdunkelung“.

- V. 10: Durch die Wortfolge „,wühlen abgrundwärts“ entstehe eine ,tonal-grammatische Unruhe“, die wieder ,weit genauer und bedrängender" als im französischen Original sei.

- V.11/12: Die Neu-Formulierung Celans „und mit ihm schwimmen, unten / und länger als sein Herz" übertreffe ,,sogar Supervielles schönste Zeile: ,et si s'arrête leur cœur...““. Celans Übersetzung, ,vollzieht das Hinabgleiten Jesu in die Tiefe sprachlich mit und ortet den impliziten Kontrast zwischen göttlicher Ewigkeit und der Kurzlebigkeit organischer Formen geheimnisvoll in Zeit und Handlung“"

Der Kommentar Steiners schließt mit dem Urteil ab, nach der Lektüre dieser Passage der Celanschen Übersetzung könne man „kaum zu Supervielle zurück“. Diese Behauptung wird zu einer verallgemeinernden, in Zitatsform im Titel des vorliegenden Aufsatzes vorzufindenden conclusio über die Grausamkeit übersetzerischer Leistungen solchen Ranges wie derjenigen Celans noch einmal gesteigert.

Steiners Darlegungsstil war in der Übersetzungswissenschaft nicht selten Gegenstand scharfer Kritik. Insbesondere an seiner weitgehend metaphorischen Sprache (vgl. u. a. Kharmandar 2018: 89) und an seiner zuweilen sexistischen Ausdrucksweise (vgl. u. a. Chamberlain 2000: 321) hat man Anstoß genommen. In der Stellungnahme Steiners zu Celan bzw. Supervielle wird ein weiteres Merkmal seines Schreibstils augenfällig, und zwar dessen gleichsam apodiktischer wie affektbeladener Duktus. Eher ungewöhnlich für den gegenwärtigen translatologischen Diskurs sind Steiners stark wertende Statements (vgl. Robinson, „The End of an Era” im vorliegenden Band). Manche sind ausgesprochen negativ wie diejenige über die „Banalität“ der Dichtung Supervielles oder die oft zitierte Bankrotterklärung an die Übersetzungsgeschichte von der Antike bis zur Neuzeit: Nach Steiner seien nämlich ,neunzig Prozent aller Übersetzungen seit Babel ohne Zweifel inadäquat, und so wird es wohl bleiben“" (Steiner/Plessner 2004: 382). Andere Kommentare sind außergewöhnlich positiv: Die Übersetzungen Celans seien 
„Geniestreiche” (Steiner/Bornhorn 2011a: 281), Rilke lasse in seinem „Höhenflug” bei der Übersetzung von Louise Labé die Möglichkeiten der Dichterin ,weit hinter sich” (Steiner/Plessner 2004: 385). Für die übersetzungswissenschaftliche Diktion dürften markante, superlativische Formulierungen wie die bereits zitierte über die schöpferisch verändernden Übersetzungen (wofür Celan exemplarisch einstehen kann) als „die grausamsten aller Huldigungen” (ebd. 2004: 387) an das Original Befremden auslösen. Steiner ist offensichtlich kein aus akademisch-wissenschaftlicher Distanz schreibender Autor. Er formuliert seine Position betont wertendemotional und stützt sie mit seiner - zweifelsfrei großen - Gelehrsamkeit, die ihn zu einer Autorität auf dem Gebiet der Vergleichenden Literaturwissenschaft und der literaturkritischen Essayistik werden ließ. ${ }^{1}$ In einem umfassenderen disziplinären Rahmen betrachtet: In der literaturwissenschaftlichen Celan-Forschung lassen sich auch andere ähnliche Schreibstile identifizieren: Bernhard Böschenstein gelangt in seinem Celan-Supervielle-Vergleich zu der Schlussfolgerung, dass „die Sprache der Übersetzung [sc. Celans] der Ort einer Raum und Zeit durchmessenen Erfahrung“" sei, während sich Supervielles Sprache „leichter, verantwortungsloser gegenüber den von ihm angewandten Mitteln” (Böschenstein 1988: 229) verhalte. Florence Pennone (2007: 322) fragt sich in ihrer Untersuchung über Celans Übersetzungspoetik, was denn an der „,bescheidenen“ Lyrik Supervielles das translatorische Interesse Celans hervorgerufen haben konnte.

In der Übersetzungswissenschaft sind solche stilistische wie affektiv zugespitzte Stellungnahmen nicht unbekannt, aber eher isolierte Erscheinungen, von denen einige markante Beispiele genannt werden sollen: In einem scharf kritischen Beitrag qualifizierte Henri Meschonnic (1972) die französischen Übersetzungen des

1 Aufschlussreich in diesem Zusammenhang wäre eine Analyse der Ausdrucksweise Steiners im Ausgang von Douglas Robinsons Theorie der Somatik, die aufzeigt, wie der Affekt die Kognition formt und leitet (vgl. Robinson 1991) bzw. von dessen späteren Arbeiten, die beide Phänomene in ihrer Spannung (nicht nur der Affekt wird zur Kognition, sondern auch die Kognition wird zum Affekt) thematisiert (vgl. bspw. Robinson 2016). 
Werks Paul Celans durch André du Bouchet, Jean Daive und Jean Pierre Burgart als „Massaker“, Jürgen von Stackelberg (2005) sah im Dichter-Übersetzer Rainer Maria Rilke einen ,übersetzerischen Egozentriker", Fritz Paepcke (zit. nach Snell-Hornby 2006: 32) steigerte sich in Wortgefechten gegen die strukturalistische Sprachwissenschaft seiner Zeit, die er als eine „Wissenschaft ohne Sprache" erachtete. Neben dem Versuch, die affektive Dimension in die wissenschaftliche Betrachtung zu integrieren (Hubscher-Davidson 2018, Koskinen 2020) und sie in ihrem Verhältnis zur Kognition zu reflektieren (vgl. bspw. Robinson 1991 und 2022 i. V.), wird aus linguistischer Warte ausdrücklich das Desiderat der Sachlichkeit in der Darstellung geäußert. Diese soll in analytischer Auseinandersetzung mit den Textvorlagen (Original wie Übersetzung), d. h. methodisch und terminologisch-deskriptiv mit dem nüchternen Instrumentarium der Sprachwissenschaft arbeitend (vgl. Stolze 1992: passim), erreicht und durch rhetorisches, literarisches sowie hermeneutisches Wissen (vgl. Kohlmayer 2019: 44-49 und 129185) verfeinert werden. Aus einer solchen Perspektive werden herausragende übersetzerische Leistungen, zu denen gewiss auch diejenige Celans gehört, nicht in schwer definierbaren Kategorien wie „Genialität“ gewürdigt, sondern als Texte, denen eine ,eigene Literarizität" (Sanmann 2013: 4) zuerkannt wird.

\section{Translatologische Analyse}

Wie lässt sich diese Identität des Translats näher bestimmen? Die Suche nach einer Antwort auf diese Frage nimmt hier ihren Ausgangspunkt bei der wesentlichen Umwertung des Status von Translaten in der gegenwärtigen Übersetzungswissenschaft. Die Übersetzung - insbesondere im Bereich der Literatur - wird zunehmend als ein „unabhängiger“ (Greiner 2004: 54-65), „eigenständiger" 'Text (Sanmann 2013: 68), als ein Produkt ,à part entière" (Lombez 2016: 18) aufgefasst, welches trotz vielfältiger Bezüge zum Original als ,,relatively autonomous from its source text“ gilt (Venuti 2019: 2). Plädiert wird dafür, die literarische Übersetzung als eigenständige Textgattung „,mit eigenem souveränen Status“ 
(Stroińska 2015: 138), ja, als ,a prototypical literary genre“ (Robinson 2017: 460) aufzufassen.

Im Lichte dieser Erkenntnisse wird hier eine differierende Art von Analyse vorgeschlagen. Diese andersartige Herangehensweise, die man translatologisch nennen kann, betrachtet das Translat als Prototext (Popovič 1976: 226), d. h. als Primärtext. Dies entspricht im Grunde der üblichen Wahrnehmung der Leser: Sie sind daran gewöhnt, einen übersetzten Text als ein Original zu lesen („Ich lese Proust.") und dabei die Präsenz des Übersetzers ,as a carrier without a substance of its own, a virtually transparent vehicle“ (Hermans 1996: 23) anzusehen. Umso mehr gilt dies für „translations that are so brilliant that they are mistaken for (or recategorized as) originals“ (Robinson 2017: 450), was Celans Übersetzung gewiss attestiert werden kann. Für die Forschung bringt dies eine Blickumkehr, eine „conversion du regard“ (Berman 1995: 65) mit sich: Die gewöhnliche Durchlaufrichtung der Untersuchung vom Original zum Translat wird abgeändert. Die Analyse fokussiert sowohl den Übersetzungstext als auch das Original zunächst einmal ,,an sich“ und führt den Vergleich zwischen den beiden Texten erst nachträglich durch. Motiviert wird dieses methodologische Vorgehen durch die Überlegung, dass die unmittelbare Nähe der Texte zueinander oft den kritischen Sinn aktiviert und Fragen der Bewertung aufkommen lässt, wie dies im Kommentar Steiners deutlich wird. Die hier vorgeschlagene Analyse verfolgt keine übersetzungskritische Zielsetzung. Sie geht vielmehr davon aus, dass das Translat anders als das Original ist, dass Differenz, Asymmetrie, Andersheit vielfach motivierte, inhärente Kategorien der Übersetzung sind. Die Stilllegung bzw. Aufhebung des kritischen Sinns soll den Blick entlasten und den Fokus auf die Texte (Translat und Original) unterstützen, um die Frage nach deren textuelle Identität klären zu können.

In der folgenden, vordergründig mit den Instrumenten der Sprachwissenschaft durchgeführten Analyse, die durch rhetorischstilistische und literarisch-poetologische Erkenntnisse zu ergänzen sein wird, soll versucht werden, in einem sachlicheren, nicht polarisierenden Duktus zunächst die Übersetzung Celans (s. Abschn. 
4.1) und dann den Originaltext (s. Abschn. 4.2) zu beschreiben. Fokussiert werden dabei insbesondere Aspekte der Informationsstruktur sowie der Lexik. Gefolgt werden die einzelnen Analysen vom Vergleich der zwei Texte miteinander (s. Abschn. 5.1) und von der Darstellung der unterschiedlichen ästhetischen Konzepte (s. Abschn. 5.2 und 5.3), die den beiden Gedichten zugrunde liegen.

\subsection{Celans "Chanson"}

Das zentrale Motiv des Gedichts „Chanson“ ist ein Gespräch mit bzw. über Jesus. Es schildert dessen allumfassende Präsenz, seine Macht und sein Wissen (Strophe 1), die in starker Opposition mit seiner Vergessenheit bzw. mit seiner zu einer bloß dekorativen Funktion mutierten Stellung (,ein Holzbild“) in der gegenwärtigen Welt (Strophe 2) stehen.

Strophe 1: Das Gedicht beginnt mit der direkten Anrede des Gesprächspartners durch Namensnennung (V1: „Jesus“) in der Vertrautheitsform „du“ (V.1), die dann in der ganzen Strophe (V. 9/11) beibehalten wird. Der Dialogpartner bleibt durchgehend in der Hörerrolle: Von ihm kommt kein Gesprächsbeitrag zurück; somit gestaltet sich der Dialog als einseitig, da er sich auf die Äußerungen des lyrischen Ich beschränkt. Drei grundlegende Charakteristika und Fähigkeiten Jesu werden im ersten Teil des Gedichts angesprochen: ,du kennst“ (V. 1), ,du kannst“ (V. 9), ,du weckst“" (V.13). Jede einzelne wird näher präzisiert.

„Du kennst“: Anhand der Affizierung des Referenz-Pronomens „sie“ im Plural durch das Summativ-Pronomen ,alle“ markiert Celan gleich zu Beginn das umfassende Wissen Jesu (V. 1: , du kennst sie alle $\left.{ }^{()}\right)$in einem informationsstrukturellen Fokus. Das pronominale Gefüge (,sie alle") wird in den nachfolgenden Zeilen kataphorisch expliziert (er kennt V. 2: „das Blatt“, V. 3: „die Wurzel“, V. 5: „die Angst“, V. 7: „das Seufzen“). Kataphora erhöhen rhetorisch die Spannung. Dazu wird durch Interpunktion (Doppelpunkt) die Aufmerksamkeit des Lesers auf jedes einzelne Element der Aufzählung erhöht. Diese Elemente werden durchgehend im Singular mit bestimmtem Artikel wiedergegeben, wodurch eine 
Abstrahierung bzw. Essentialisierung (im Sinne von das Blatt, die Wurzel, die Angst, das Seufzen, die symbolisch sowohl für die Natur als auch für die Lebewesen stehen) erzielt wird. [Für Steiner machen diese Singulare die Anrufung Jesu ,erschütternd unmittelbar".] Jeder Bestandteil wird zweimal durch satzförmige bzw. weitere zwei Male durch attributive Gefüge näher beschrieben, wodurch eine doppelte formale Symmetrie entsteht: Dem „Blatt“ folgt ein Relativsatz (V. 2: „das Waldgrün bringt") mit einem starken visuellen Effekt. Die „Wurzel“ wird ebenfalls von einem Relativsatz begleitet, in dem das nominalisierte Superlativum (V. 3: ,ihr Tiefstes") ein einprägsames Bild kreiert [Steiner sprach an dieser Stelle von der doppelten - abstrakten wie bildlichen - Genauigkeit der Steigerungsform] und die Verbpräfixe (V.4: „aufsammelt“, „vertrink $\mathrm{t}^{\text {") }}$ die Gegensätzlichkeit und Spannung der beiden verbalen Aktionen effektvoll auffangen. Die „Angst“ wird postdeterminiert durch ein Genitiv-Attribut (V. 5: ,des Taggeschöpfes“) [das Steiner als eine „Engführung“, d. h. als eine lexikalische Neuschöpfung Celans ansieht]. Dieses nominale Kompositum ist ein Archaismus, der im Woerterbuch der deutschen Sprache von 1810 als „ein Geschöpf, das nur einen Tag lebt, an einem Tage geboren wird und stirbt" (Campe 1810: 768) definiert wird. Der nachfolgende temporale Nebensatz präzisiert den Auslöser der „Angst“ jenes Lebewesens: Es ist die aufbrechende Nacht (V. 6: „wenn es sich nachthin neigt ${ }^{(\bullet)}$, die ihrer kurzzeitigen - eintägigen - Existenz ein Ende setzt; jene Angst ist die Angst vor dem Tode. Semantisch auffällig ist an dieser Stelle das Lexem ,nachthin“ mit einem starken visuellen und poetischen Effekt: Der Tag neigt sich zur Nacht hin. Das Richtungsadverbialkompositum ,nachthin“ ist ein von Celan kreierter Neologismus [auch Steiner sieht darin ein Celan eigentümliches Wort, das ,vor ihm nicht da gewesen“ ist], in dem er zu einem spezifischen Mittel seines eigenen poetischen Stils (Suffixbildungen mit -bin, -her, -wärts) greift (vgl. Olschner 1985: 113f.), um „den prozessualen Charakter" (Böschenstein 1984: 87) der Aktion hervorzuheben. Das „Seufzen“ als das vierte und letzte Element der Aufzählung ist genauso wie das vorige von einem Genitiv-Attribut begleitet (V. 7: , das Seufzen dieser Erde ${ }^{\circ}$ ), wodurch die formale Sym- 
metrie unterstützt wird. Die Verortung dieses Elements „im Raum“ (V.8) wird durch den Relativsatz (V. 8: „der sie umschweigt") noch präziser. Hier fällt ebenfalls eine lexikalische Neuschöpfung Celans [von Steiner auch als solche identifiziert] auf, und zwar das mit um- präfigierte Verb „schweigen“. Dadurch kreiert Celan ein Bild von großer Poetizität, in dem Visuelles (der die Erde umfassende Raum) und Auditives (Schweigen als privatives auditives Phänomen) ineinander verschmelzen (um-schweigen).

„Du kannst“: Im mittleren Teil der ersten Strophe beschreibt das lyrische Ich die außergewöhnlichen Fähigkeiten Jesu mittels dreier modalen Klammern (V. 9: ,du kannst [...] begleiten“; V. 10: ,[...] dich wühlen“; V. 11: ,[...] schwimmen“). Diese Passage ist visuell geprägt. Die Bilder zeichnen die Kapazität Jesu, in die marine Unterwelt hinabzusteigen, und zwar immer tiefer (V. 10: „dich wühlen abgrundwärts") bis zum Meeresgrund (V. 11: „schwimmen, unten"), wo selbst ein Meeresbewohner der Lebensgefahr (V. 12: „länger als sein Herz') ausgesetzt ist. Syntaktisch auffällig sind die Ausklammerungen in drei aufeinanderfolgenden Verszeilen (V. 10/V. 11/V. 12), in denen das Direktional-, das Positionsund das Temporaladverb mit nachfolgendem Vergleich sich alle außerhalb der modalen Verbalklammer im Nachfeld befinden (,kannst [...] wühlen abgrundwärts“ / „schwimmen, unten“ / „und länger als sein Herz"). Ergänzende Informationen im Nachfeld, die üblicherweise eine Marke der Mündlichkeit darstellen, sorgen in einem dichterischen Text umso mehr für Auffälligkeit. Besondere poetische Ausdruckskraft hat vor allem die letzte Zeitangabe „länger als sein Herz" (d. h. des begleiteten Fisches). [Steiner sieht darin einen Kunstgriff Celans, durch den er ,den impliziten Kontrast zwischen göttlicher Ewigkeit und der Kurzlebigkeit organischer Formen geheimnisvoll“" zur Sprache bringe.]

„Du weckst“: Die Schlusspartie dieser Strophe fokussiert die Handlungsmächtigkeit Jesu, die - anders als bei „kennen“ und „können“ - durch ein Handlungsverb (V. 13: „du weckst”) dargestellt wird. Das Objekt, auf das dieses transitive Verb ausgreift und es somit seinem Verfügungsbereich angliedert, ist - analog zu V. 2/3/5/7 - ein Nomen mit bestimmtem Artikel im Singular 
(V. 13: „das Lied“), wodurch gleichfalls eine Abstrahierung bzw. Essentialisierung erwirkt wird. Dieses Lied, das titelgebend („Chanson") ist, wird durch ein Präpositionalobjekt (V. 13/14: ,von jenseits der Meere“) in seiner Herkunft näher umrissen. Der darauffolgende Satz (V. 14: „dass es lebt") steht ebenfalls in Korrelation mit dem hier zentralen Verb ,wecken“. Zwischen der Bedeutung der Basis (V. 13: „du weckst”) und der Bedeutung des Adjunkts (V14: „es lebt”) besteht ein semantischer Zusammenhang im Sinne der Folge: Es ist ein Wecken zum Leben. Zu vermerken ist hier der Kontrast, der sich zwischen der Kurzlebigkeit jenes von Todesangst beherrschten „Taggeschöpfes“ (V. 5) und dem durch die Weckhandlung Jesu ermöglichten Leben entspinnt. [Dies hat in manchen Studien - bspw. in Böschenstein (1988: 228) - zu einer theologischen Interpretation der Stelle geführt.] In V. 15 bewirkt das Deiktikon „die“ („die's nun singt“), das informationsstrukturell ein neues Thema einführt, eine Bündelung der Aufmerksamkeit, die umso größer ist, als die nominale Vorinformation, die üblicherweise einem Demonstrativum im Text vorausgeht und seine $\mathrm{Zu}-$ ordnung zu einer Person oder Sache ermöglicht, hier fehlt. Eine partielle Auflösung dieser Ungewissheit erfolgt durch das Genus: Das Femininum „die“ lässt auf eine weibliche Person schließen. Deren Handlung (V. 15: ,und die's nun singt, muss singen“) ist ebenfalls stark markiert, und zwar zweifach: rhetorisch durch die Wiederholung des Verbs und semantisch durch die Modalisierung der Prädikation mithilfe des Verbs „müssen“, das einen Zwang, ein Gebot bezeichnet. Die tiefe Wirkung des Lieds auf die singende Person wird in zwei Verben mit derselben Vorsilbe (V. 16: „durchzittert und durchbebt" $)$ zum Ausdruck gebracht. Die Wiederholung des Präfixes durch- (anstatt der möglichen Elidierung beim zweiten Verb) übt rhetorisch eine Fokusfunktion als nähere Beschreibung des unbekannten neuen Themas „die“ aus. [Ebenfalls $\mathrm{zu}$ vermerken ist die aus der literaturwissenschaftlichen Forschung bekannte Vorliebe Celans für die Vorsilbe durch- als eine „Ausdrucksmöglichkeit für die betont durchdringende Eigenschaft eines Phänomens“ (Olschner 1985: 80).] 
Strophe 2 beginnt ebenfalls mit einer direkten Anrede, jedoch dieses Mal an eine unbekannte Person, die zu einem genauen Hinhören auf Zukünftiges angehalten wird (V.17: „hör gut zu morgen“). Diese neue zeitliche Verortung bringt eine neue Bezugsordnung mit sich: Vom bisherigen „du“ (Hörerrolle) ist nun Jesus zu dem von Distanz gekennzeichneten ,er“ (Referenzrolle) mutiert. Diese im Zeichen der Ferne stehende Transformation wird zweifach durch die Wiederholung des Pronomens und unter Hinzunahme der Partikel „selber“ (V. 17/18: „denn er, / er selber“) zusätzlich markiert. In dieser kurzen Strophe werden in konzentrierter Form alle drei eingangs skizzierten besonderen Fähigkeiten Jesu der Reihe nach kontrastiert, ja annulliert: sein früheres allumfassendes Wissen (V. 1) weicht nun der Wissensvergessenheit (V. 18: ,er selber weiß es nicht mehr $^{6}$ ); seine lebensspendende Kapazität (V. 13/14) erlischt in der Wahrnehmung Jesu als totes Holzstück, dem nun eine bloß dekorative Funktion als Bild zukommt (V. 19: „ein Holzbild, so seht ihr ihn“); seine Omnipräsenz, die ihn die Untiefen ergründen und bis an Orte durchdringen lassen, die anderen Lebewesen verwehrt bleiben (V. 9/10/11/12), entwickelt sich zu Starre und Immobilität in einem banalen häuslichen Umfeld (V. 20: „dastehen auf dem Kamin“). Die Kontraste werden auch informationsstrukturell durch die Spitzenstellung des rhematischen nominalen Kompositums (V. 19: „ein Holzbild, so seht ihr ihn”) sowie durch die Versetzung der Lokalangabe ins Nachfeld des Verbs (V. 20: „dastehen auf dem Kamin“) unterstützt. Schließlich besetzt Jesus nicht mehr die Handlungsrolle wie in der ersten Strophe (V. 1: ,du kennst“, V. 9: ,du kannst“, V. 13: „du weckst“), sondern ist Objekt der Handlung (genauer: Direkt-Objekt im Akkusativ), ja der bloßen Betrachtung (V. 19: ,so seht ihr ihn“). Auffällig ist ebenfalls die Änderung des Adressaten: Das lyrische Ich wendet sich zunächst an eine Einzelperson (das im Imperativum implizite „du“ in V. 17: ,hör gut zu“), dann an eine Mehrzahl von Personen (V. 19: ,so seht ihr ihn“). Diese völlig neue Wahrnehmung Jesu steht somit nicht mehr für ein isoliertes Phänomen, sondern wird repräsentativ für größere Menschenmengen. Dadurch bezeichnet Celan die Gottferne der Menschen. 


\subsection{Supervielles „Chanson“}

Das Gedicht „Chanson“ entstammt dem Band Gravitations, der Texte umfasst, die Supervielle zwischen 1922 und 1925 geschrieben hat. Somit gehört es zu seinem Frühwerk. Das Gedicht stellt zwei unterschiedliche Bilder Jesu gegenüber: einerseits seine Allgegenwärtigkeit und Mächtigkeit in der Schöpfung, andererseits seine Schrumpfung zu einem bloßen Gegenstand der Devotion (,une statue peinte") in häuslichem Milieu.

Strophe 1: Das Gedicht beginnt mit einer Anrede. Der Gesprächskontakt wird durch die Namensnennung (V.1: ,Jésus") hergestellt, das Gespräch selber wird dann in der ganzen Strophe im Modus der Vertrautheit (V. 1: „tu sais“, V. 9: ,tu peux“, V. 13: „tu fais naittre") geführt. Um diese drei Verben herum wird der erste Teil des Gedichts konstruiert, indem sie näher beschrieben werden.

Dem ersten Verb (V. 1: „tu sais") werden vier Phänomene subsumiert, wobei deren Aufzählung zunächst Naturelemente (V. 1: „feuille“, V. 3: „racines"), dann Emotionen (V. 5: „terreur“, V. 7: ,soupir") umfasst. Die Beschreibung dieser Elemente erfolgt symmetrisch: die ersten zwei durch Relativsätze (V. 1/2: „chaque feuille / qui verdira la fôret“, V. 3/4: ,les racines qui recueillent / et dévorent leur secret"), die beiden letzten durch Genitiv-Attribute mit Zuordnungsfunktion (V. 5: ,la terreur de l'éphémère“, V. 7: „le soupir de la Terre“). „Feuille” in der Einzahl ist von dem KodeArtikel „chaque“ mit den semantischen Merkmalen \{Ganzheit\} und \{bestimmend\} begleitet, die somit das Wissen Jesu als umfassend und zugleich präzise darstellen. Dieses Wissen um jedes Blatt erweist sich ebenfalls als vorausschauend: Die Futurform des Verbs (V. 2: „qui verdira la fôret") verweist auf das Vorwissen Jesu um Zukünftiges, um das Werden jenes Blattes. Das nächste Element in der Aufzählung steht in der Mehrzahl (V.3: „les racines") und wird durch zwei Verben beschrieben, die gegensätzliche Aktionen (V. 3/4: „recueillent / et dévorent") auf deren semantisch nicht aufgeschlüsseltes Objekt (V. 4: „leur secret") ausüben. [Für Steiner wirkt das zweite Verb „dévorer“ „zufällig oder auch nur sonor"..] Der dritte Bestandteil der Aufzählung (V. 5: „,la terreur") 
weist auf eine tiefe negative Empfindung vor dem Hintergrund der Vergänglichkeit hin, für die das essentialisierende Abstraktum (V. 5: „de l'éphémère“) steht. [Steiner schätzt „l'éphémère“ als „schweifend unheimlich“ ein.] Die anschließende Temporalangabe (V. 6: „à l'approche de la nuit") präzisiert die näheren Umstände (die hereinbrechende Nacht), die jenen Terror auslösen. Die Aufzählung schließt mit einer auditiv geprägten Szenerie in der Präsentation der Manifestation einer weiteren tiefen Emotion (V. 7: „le soupir") ab, die durch die Großschreibung des Bezugsnomens (V. 7: „de la Terre") auf ein allumfassendes Phänomen hinweist. $\mathrm{Zu}$ vermerken sind an dieser Stelle die unterschiedlichen Hörregister in ,soupir“ (als auditiv vernehmbares Phänomen) und ,silence“ (als privative auditive Erscheinung). Das Adjektivum ,infini“ verstärkt die Wirkung des Nomens ,silence“ (V. 8) in zweifacher Hinsicht, und zwar sowohl in seiner zeitlichen Dimension als Unendlichkeit (d. h. als ewige Stille) als auch in seiner superlativischen Intensität (als tiefe Stille). [Auf Steiner wirkt ,infini” überraschenderweise ,abgeschwächt“.]

Der mittlere Teil der ersten Strophe baut auf dem Verb „tu peux“" auf. Beschrieben wird die Fähigkeit Jesu, Fische in die bzw. in der Meerestiefe zu begleiten, wo diese durch ihre Betriebsamkeit (V. 11: ,quand ils tournent et retournent") Unruhe (V. 10: ,tourmentant les profondeurs") stiften. Deren Getriebe wird rhetorisch durch die Wiederholung des Verbs unterstützt, wobei die re-Präfigierung beim zweiten Verb (V. 11: „,tournent et retournent") sowie die gleich doppelte Wiederkehr des Bausteins ,-tour-“ (V. 10-11) den visuellen sowie auditiven Effekt noch einmal verstärken. Die Begleitung der Fische durch Jesus wird selbst nach deren Tod fortgesetzt, der im nachfolgenden Konditional-Satz (V. 12: „et si s'arrête leur cœur") poetisch umschrieben wird. [Steiner empfand ihn als „Supervielles schönste Zeile“.]

Im Fokus der Schlusspartie dieser Strophe steht die dritte Fähigkeit bzw. Aktion Jesu (V. 13: ,tu fais naittre“). Dieses zweiwertige Verb mit der Bedeutung ,lassen, veranlassen“ zeigt, dass die Handlungsinitiative zwar beim Subjekt (V. 13: „tu“, d. h. Jesus) liegt, aber die Aktion selber von einer anderen, hier nicht näher prä- 
zisierten Person durchgeführt wird. Das Objekt (V. 13: „des chansons") nennt das Ergebnis der Handlung: Geboren werden Lieder, im Plural und vom partitiven Artikel „des“ begleitet, der auf die Nachinformationen (V. 14/15/16) verweist: Durch die Lokalangabe wird der Ort der Entstehung jener Lieder (V. 14: „si loin au delà des mers") genannt, dessen überraschende Ferne durch das Quantitativ-Morphem „si“ beim Adverb „loin“ markiert wird. Genannt wird ebenfalls die Wirkung jener Lieder auf die singende Person. Deren Intensität löst in der Singenden eine grundtiefe physische Reaktion aus (V. 15/16: „que la fille qui les chante / en tremble au fond de sa chair"). Das prägnante Bild des in seinem Innersten zitternden Mädchens macht die starke Rührung, die jene Lieder in ihr bewirken, anschaulich.

Strophe 2: Der finale Gedichtteil beginnt mit einer imperativisch formulierten, durch ein Intensitätsadverb markierten Aufforderung in der Mehrzahl, also an eine größere Menschenmenge adressiert (V. 17: „écoutez-le bien“). Jesus, das „du“ der ersten Strophe, wird hier in die Referenzrolle (,le“) versetzt. Der Ersatz durch den Artikel hat sowohl anaphorische (verweist auf den in V. 1 genannten Namen) als auch kataphorische Funktion (antizipiert den gleich in V.18 erneuten Namensnennung: „Jésus aura oubliéc"). Diese eindringlich formulierte Aufforderung, Jesus jetzt gut zuzuhören, wird durch geänderte Umstände in der Zukunft (angezeigt durch das Temporaladverb in V. 17: ,demain“" sowie durch die Futurform der beiden nachfolgenden Verben in V. 18: ,aura oublié“ bzw. V. 19: „,ne sera“') begründet. Eine starke Opposition zur ersten Strophe des Gedichts entspinnt sich in diesem Schlussteil: Der allwissende Jesus (V. 1/3/5/7: ,tu sais chaque feuille [...] / les racines [...] / la terreur [...] / et le soupir [...]") wird der Vergesslichkeit anheimfallen (V. 18: "Jésus aura oublié"), sein bislang durch herausragende Fähigkeiten (V.9-12, V.13-16) gesicherter Status wird künftig auf nur ein Merkmal bzw. nur eine Rolle - eine bemalte Statue, ein Objekt der häuslichen Devotion - reduziert sein. Die eingeschränkte Negation ,ne [...] que“ bildet durch die suggerierte Ausschließlichkeit einen informationsstrukturellen Fokus auf das 
genannte Charakteristikum (V. 19/20: „ne sera qu'une statue / peinte sur la chéminée").

Die hier dargestellte Art von Analyse erhebt keineswegs den Anspruch auf Vollständigkeit: Sie kann und möchte nicht ,alles“ über die beiden zur Debatte stehenden Texte ausgesagt haben; Weiterführungen im Rahmen der literarischen Hermeneutik und der Celan- bzw. Supervielle-Forschung mit Blick auf die Poetik der beiden Dichter, auf deren jeweiligen Stil, auf die Interpretation der beiden Gedichte sind erforderlich, um den Texten und ihren Autoren angemessen gerecht zu werden. Die translatologische Analyse versteht sich vordergründig als eine Diskussionsgrundlage, die es erlaubt, (a) in einem sachlichen Duktus die Texte in ihrer sprachlichen Verfassung zu beschreiben, (b) den Blick des Lesers für die filigrane Struktur des jeweiligen Textes zu schärfen, auf der letztlich dessen Individualität (d. h. ,Identität ${ }^{\circ}$ ) und Poetizität beruht, (c) die unterschiedlichen Ebenen des wissenschaftlichen Diskurses (Text, Interpretation, Kritik) auseinanderzuhalten, (d) eine Basis für intersubjektive Nachvollziehbarkeit der Bewertungen zu schaffen. In Steiners Kommentar zu „Chanson“ gehen diese Aspekte oft undifferenziert ineinander. Dies kann beispielsweise an seiner Anmerkung zu dem Passus V. 7/8 („Et le soupir de la Terre / Dans le silence infini“ bzw. „Das Seufzen dieser Erde / im Raum, der sie umschweigt") veranschaulicht werden:

Über Supervielle weit hinausgreifend [= Kritik, L. C.], erfüllt die Übersetzung [= Text, L. C.] die Intention von Schwere und Verdunkelung [= Interpretation, L. C.] (was im Original abgeschwächt ist durch , infini“) [= Kritik, L. C.]. (Steiner/Plessner 2004: 387)

Nachfragen drängen sich auf: Wirkt ,infini“ mit seiner doppelten Semantik (als , unendlich“ und ,tief") im Original (V. 8) in der Tat „abgeschwächt"? Des Weiteren: Inwieweit ist „dévorer" bei Supervielle (V. 4) ,zufällig, oder auch nur sonor“, wo diesem Lexem eine gegensatzbildende Funktion im Binom ,recueillent et dévorent“ (V. 3/4) zukommt? Ebenfalls: Ist das Abstraktum „l'éphémère“ als Inbegriff des von ,terreur“ geplagten Vergänglichen (V. 5) tatsächlich ,schweifend unheimlich“? Vor dem Hintergrund der textuellen Grundlage bzw. Analyse leuchten diese Einschätzungen nicht 
unmittelbar ein. Schauen wir uns nun die Auffassung Steiners über die Relation zwischen Translat und Original, die seinem Celan-Supervielle-Kommentar zugrunde liegt, näher an.

\section{Das Verhältnis von Übersetzung und Original}

Im hermeneutischen Vorgang zwischensprachlichen Übersetzens identifiziert Steiner bekanntlich vier Phasen: Vertrauen, Aggression, Einverleibung und Reziprozität. Die Bezeichnung dieser aufeinander folgenden Phasen, deren Gesamtheit Steiner als hermeneutic motion definiert, lässt leicht einsehen, dass er damit keinen streng theoretischen Anspruch erhebt. Sein Anliegen ist es, den Übersetzungsakt von der Praxis her zu beschreiben. Zentral für Steiner sind weniger die linguistischen Mechanismen des Transfers von einer Sprache in eine andere; im Vordergrund steht für ihn die Dynamik des Übertragungsprozesses und seine emotionale Beladung. Dieser hermeneutische Vorgang lässt sich schließlich wie eine ,anatomy of feelings, not to say passions, underlying the process of ,understanding““ (Heidelberger-Leonard 1985: 22) lesen.

Von besonderem Interesse für die vorliegende Studie ist insbesondere die 4. Phase. Das fundamentale Ungleichgewicht der ersten drei Schritte ist laut Steiner im Versuch der Schaffung von Reziprozität auszugleichen: Dem Original muss ersetzt werden, was es durch Übertragung verloren hat und so hat die Übersetzung die Aufgabe, dem Original dazu zu verhelfen, seine ursprüngliche Statur erkennbar zu machen. Der durch Übersetzung ausgeplünderte Originaltext muss durch das von der Übersetzung mitgebrachte Fortbestehen und Weiterwirken sein Prestige wiedererlangen und sogar zum weiteren Wachstum gebracht werden:

Kein Zweifel: Echo bereichert, ist mehr als Schatten und lebloses Abbild. Wie ein Spiegel reflektiert die Übersetzung nicht nur, sie leuchtet auch selbst. Der Originaltext gewinnt durch verschiedene Verhältnisse und Abstände zwischen ihm und seinen Übersetzungen. Die Reziprozität ist dialektisch: Abstand und Nähe verändern das ,Format ${ }^{\star}$ der Signifikanz. Manche Übersetzungen lassen uns von der Leinwand zurücktreten, andere stellen uns dicht vor sie hin. (Steiner/Plessner 2004: 317) 
Übersetzungen verhelfen Originaltexten zu größerer Überlebensdauer und zu einer geographischen und kulturellen Breitenwirkung, die sie ohne Übersetzungen nicht gehabt hätten. (Supervielle ist nach wie vor relativ wenig ins Deutsche übersetzt worden. Celan gilt mit seiner Gedichtauswahl immer noch als der bedeutendste Übersetzer Supervielles im deutschen Sprachraum.) Translate können auch die Bedeutung eines Werkes, die in der heimatlichen Kultur unterschätzt oder übersehen worden war, erst offenbaren. Die Prägnanz eines Originals tritt folglich dialektisch im Vergleich mit seiner Übersetzung und durch Übersetzung besser in den Vordergrund. Wenn die Übersetzung qualitativ hinter dem Ausgangstext zurückbleibt, werden dessen Vorzüge umso deutlicher. Wenn sie hingegen das Original qualitativ (z. B. auf stilistischer Ebene oder durch die Freilegung neuer Interpretationsmöglichkeiten) übertrifft, weist sie darauf hin, dass darin Sinnpotenzialitäten vorhanden waren, die es selbst noch nicht zum Erscheinen gebracht hat und die erst durch Übersetzung zutage gefördert werden. Diesen letzten Fall illustriert - so geht es aus dem bereits zitierten Kommentar Steiners hervor - die Übersetzung Celans geradezu exemplarisch. Durch ihre eigene Prägnanz stellt sie aus seiner Sicht das Original stark in den Schatten.

Die Relation zwischen ausgangs- und zielsprachlichem Text wird gegenwärtig unter einer differenten Perspektive und in anderen Termini reflektiert. Diese Überlegungen gehen über die herkömmliche originalzentrierte Sichtweise wie u. a. bei Steiner hinaus bzw. verlegen den Fokus vielmehr auf das Translat. Die bereits erwähnte Umdefinierung dieses Verhältnisses, die das übersetzerische Produkt aufwertet, ja als autonomen Text erfasst, führte neuerdings zur Attribuierung des Prädikats „Werk“ ebenfalls an (literarische) Übersetzungen. Diese Eigenschaft, die traditionell ausschließlich dem Original zuerkannt wurde, wird nun auch dem Translat zugeschrieben. Erste Impulse für die Auffassung der literarischen Übersetzung als ästhetisches Objekt und somit als Kunstwerk stammen von Norbert Greiner (2004: 22). Diese Auffassung beleuchtet er mit der These der „ontologischen Differenz“ (Greiner 2004: 15) zwischen literarischen und pragmatischen Texten, die 
er mit zwei Argumenten begründet: (1) Fiktionale Äußerungen stellen weder einen Kommunikationsakt dar noch sind sie an einen Empfänger gerichtet. In Sprachkunstwerken bildet die Sprache eine sich selbst genügende Sinnstruktur. (2) Die formalen Elemente der Sprache wie Lautqualität, Rhythmus, Klangfarben, Metrum u. a. sind nicht Beiwerk, sondern wirken sinnkonstituierend. Würde man sie verändern, würde der Sinn verändert: In literarischen Texten ist die Form semantisiert. Angesichts ihrer spezifischen Seinsweise, Erfahrbarkeit und Wirkweise plädiert Greiner (2004: 17) für die These der „Autonomie“ literarischer Texte. Stark vertreten und gefördert wird der Gedanke der literarischen Übersetzung als Werk u. a. in der Germersheimer Übersetzerforschung (vgl. Tashinskiy 2016).

Die Idee der Eigenständigkeit der literarischen Übersetzung, die - als (Kunst-)Werk - über eine „eigene Identität“ verfügt, wird durch Celans Übersetzung vom Gedicht „Chanson“ vortrefflich illustriert. Diese These soll nun durch den Vergleich von Translat und Original argumentiert werden.

\subsection{Die Identität des Translats}

Celan hat das Gedicht „Chanson“ am 1. und 2. Juni 1960 unter dem Eindruck des Todes von Supervielle im Mai 1960 übersetzt. Das Entstehungsdatum der Übersetzung ist in seinem Exemplar von Gravitations (1925) notiert. Die Wahl dieses Gedichts, in dem Jesus als ein stummes Holzbild auf dem Kamin beschrieben wird, soll laut literaturwissenschaftlichen Studien auf eine Diskussion Celans über Gott mit Nelly Sachs in Zürich zurückgehen bzw. dadurch beeinflusst worden sein. Diese Diskussion sei in das von Celan am 30. Mai 1960 verfasste Gedicht „Zürich, Zum Storchen“, in dem Gott die zentrale Figur darstellt, ebenfalls eingegangen (vgl. Pennone 2007: 326f.).

Anhand der auf mikrostruktureller Ebene durchgeführten Analyse des Gedichts „Chanson“ wird deutlich, dass die Übersetzung Celans eine stark durchgearbeitete Struktur aufweist, die auch genetisch durch den Vergleich der insgesamt sechs, im Celan-Ar- 
chiv vorzufindenden Varianten dieser Übersetzung (vgl. Böschenstein 1988) zurückverfolgt werden kann. Die intensive Arbeit Celans am Translat zeugt unmittelbar davon, dass er sich bei jedem Vers in einem profunden und vielseitigen Dialog mit dem Originalautor und -text befindet. In diesem dichterischen Gespräch bedient er sich verschiedener Strategien der (1) Abbildung und Kompensation, (2) Abstrahierung, (3) informationsstrukturellen Fokussierung, (4) Transformation der originalen Bildlichkeit sowie der (5) eigenen stilistischen Prägung.

(1) Celan ist darum bemüht, dem Original nahe zu bleiben, indem er strukturelle Abbildungen (die Symmetrie der Relativsätze bzw. Genitivattribute in V. 2-8) kreiert und nach Kompensationsmöglichkeiten sucht, wenn der deutschen Sprache Mittel fehlen, die in der französischen vorliegen (V. 2: Transitivität des Verbs ,,verdir““ vs. „Blattgrün bringt") bzw. anders als im Französischen besetzt sind (V. 7: Großschreibung von ,la Terre“ vs. Spezifizierung durch das Demonstrativum in ,dieser Erde“).

(2) Durch den ausnahmslosen Gebrauch des Singulars unterzieht Celan alle nominalen Elemente im Gedicht einer Abstrahierung bzw. Essentialisierung. Während das Original eine Variabilität des Numerus und der Artikel-Determinanten (V. 1: ,chaque feuille“, V. 3: „les racines“, V. 5: „la terreur“, V. 7: „le soupir“, V. 9: „les poissons", V. 13: ,des chansons“) aufweist, präsentiert das Translat Konsequenz in der durchgehenden Beibehaltung der Einzahl und des bestimmten Artikels (V. 2: ,das Blatt“, V. 3: ,die Wurzel“, V. 5: „die Angst“, V. 7: „das Seufzen“, V. 9: „den Fisch“, V. 13: „das Lied“). Mittels dieses am Wesenskern der Dinge und Phänomene orientierten Blicks dringt Celans Text tief ins Bewusstsein des Lesers, die Übersetzung wirkt durchgreifend. [Steiner nannte diese Wirkung ,erschütternd unmittelbar”.]

(3) Starke Brüche zum Vorlagetext markiert die Übersetzung vornehmlich auf der Ebene der Informationsstruktur. Der französische Vorlagetext ist durch einen fließenden unmarkierten narrativen Ton gekennzeichnet; die Aufmerksamkeit des deutschen Lesers wird hingegen vom Übersetzer Celan durch unterschiedliche Strategien der Fokussierung wirkungsvoll auf einzelne Elemente ge- 
lenkt: Die Katapher und der Doppelpunkt (V. 1: „du kennst sie alle:") haben die Funktion, wichtige Informationen anzukündigen und somit Aufmerksamkeit zu erwecken. Diese Wirkung wird ebenfalls durch eine besondere Wortstellung erzielt: An mehreren Stellen werden einzelne Elemente ins Nachfeld des Verbs versetzt (V. 10: „dich wühlen abgrundwärts“, V. 11: „, mit ihm schwimmen, unten“, V. 17: „hör gut zu morgen“, V. 20: „dastehn auf dem Kamin“). Die Übersetzung präsentiert augenfällige Kontraste (die Opposition Tag-Nacht in V. 5: „Taggeschöpf“ vs. V. 6: „nachthin'), die im Original weniger deutlich sind bzw. erst kognitiv nachzuvollziehen sind (V. 5: „éphémère“ vs. V. 6: „,nuit"). Die rhetorische Fokusfunktion der Wiederholung, durch die das repetierte Element an informatorischer Gewichtung gewinnt, wird in der Übersetzung intensiver als im französischen Ausgangstext ausgewertet (V. 10: „tourmentant“, V. 11: ,tournent et retournent” bzw. V. 15: „die’s nun singt, muss singen“, V. 16: „durchzittert und durchbebt“, V. 17/18: „er, / er selber"). Das Translat ambiguiert durch unaufgeschlüsselte Deiktika und belässt somit den Leser in Aufmerksamkeit reizender informatorischer Unklarheit (V. 15: „die's nun singt"), wo das französische Lesepublikum eine deutliche nominale Bezeichnung (V. 15: ,la fille qui les chante") präsentiert bekommt. [Steiner wertet unter dem hier geschilderten Aspekt das Original als „wohlgestaltet, aber nicht frei von Banalitäten und Stereotypen“ und zertifiziert der Übersetzung Genialität: Celan „verdichtet und versteigert, wie es wohl nur bei seinem Genie möglich war.']

(4) Autonomie weist das Translat gleichfalls durch erhebliche Änderungen der Bildlichkeit nach, die als individuelle Lesarten Celans anzusehen sind. Das fr. „secret“ (V.4) mit den semantischen Hauptmerkmalen ,nur Eingeweihten Bekanntes“ bzw. „Unerforschtes, Unerforschbares“ wird im deutschen Text zu „Tiefstes“ (V. 3), das differente Assoziationen (visuell als tiefster Punkt in der Erde bzw. als zum Innersten, zum Kernwesen der Wurzel gehörend) auslöst. Die starke Intensität des fr. Lexems ,terreur“ (V. 5) wird in der Übersetzung durch „Angst“ (V. 5) abgeschwächt. Das allgemein gehaltene fr. „éphémère“ (V. 5) wird bei Celan durch 
„Taggeschöpf“ (V. 5) konkret. Supervielles semantisch mehrwertiges „silence infini“ (V. 8) wird im deutschen Text als „schweigen“ (V. 8) monosemiert. Das expressive Bild der hin und her ihre Bahnen ziehenden Fischschwärme in der fr. Textvorlage (V. 11: ,ils tournent et retournent") ist im Translat visuell weniger einprägsam (V. 11: ,schwimmen“) bzw. die Übersetzung rückt vielmehr die Tiefe in den Mittelpunkt der Aufmerksamkeit (V.11: „mit ihm schwimmen, unten"). Wo Supervielle einen Endpunkt des Lebens der Fische (V. 12: ,,si s'arrête leur cœur") nennt, umschreibt Celan und verlegt den Fokus auf die Transzendierung jenes Endes (V. 12: „länger als sein Herz"). Am augenfälligsten ist die Änderung des zentralen Bildes im Schlussteil des Gedichts: Der französische Leser sieht vor seinen inneren Augen eine „statue peinte“ (V. 19/20), der deutsche „ein Holzbild“ (V. 19). An dieser Stelle ist ebenfalls eine gewichtige Änderung des verbalen Tempora-Regimes zu verzeichnen: Durch die Futurform der beiden Verben präsentiert Supervielle die Gottvergessenheit als eine Zukunftsvision (V. 18/19: "Jésus aura oublié / Ne sera qu'une statue"), die Übersetzung hingegen durch das Präsens als eine bereits vorhandene Realität (V. 18: „er selber weiß es nicht mehr", V. 19: ,,so seht ihr ihn“), von der das lyrische Ich im Translat - wiederum anders als im Original sich durch einen Zusatz (V. 19: ,,so seht ihr ihn”) deutlich distanziert: Der Sprechende (im Grunde: der Dichter-Übersetzer Celan) teilt die Wahrnehmung Gottes als ein Holzbild mit der angesprochenen Menschenmenge (,ihr") nicht: Er beschreibt bloß die Gottesferne der Menschen, wo Supervielle eine radikalere theologische Aussage macht: Die Welt ist gottlos. Diese zwei letzten aufschlussreichen Differenzen im Schlussteil der zwei Texte ziehen tiefgreifende Konsequenzen nach sich, die hermeneutisch bzw. poetischpoetologisch auf beiden Seiten (in der Dichtung Supervielles und derjenigen Celans) weiter zu reflektieren sind. [Mit der Ausnahme von V. 12, die Steiner als „Supervielles schönste Zeile“ ansieht, schätzt er die originalen Bilder als ,,schweifend“, ,abgeschwächt“ und „konventionell“ ein, Celans hingegen als ,genauer“ bzw. als „über Supervielle weit hinausgreifend“.] 
(5) Celan lässt seine eigene dichterische Stimme an mehreren Stellen im Translat deutlich hören. Wo Supervielle ein Neologismus (V. 5: „l'éphémère“) bringt, macht Celan sein ausgeprägtes Interesse für archaische Wörter geltend (vgl. Seng 2007: 29) (hier durch die Verwendung von „Taggeschöpf“ in V. 5). Bekannt aus der Celan-Forschung ist der Umstand, dass er eine „Wachheit für Wortgeschichte mit entlegenen oder veralteten etymologischen Nuancen" (Olschner 1985: 47) an den Tag legte, indem er manche archaischen Wörter wiederbelebte. Celan exzipierte oft aus den Werken anderer Dichter (z. B. Wolfgang Borchardt) untergegangene, teilweise aus dem Spätmittelalterlichen übernommene Wörter, die sich nur noch in alten Nachschlagewerken (hier: in einem Wörterbuch von 1810) finden. Celans eigentümliche Vokabeln und lexikalische Präferenzen, die Gegenstand zahlreicher literaturwissenschaftlicher Untersuchungen sind, lassen sich im Translat nachweisen: Seine Vorliebe für „Tiefstes“ (Olschner 1985: 86f.) (hier: V. 3), ,schweigen“ (ebd.: 133ff.) (hier: V. 8), für die Vorsilbe „durch-“ (ebd.: 80) (hier: V. 16), für Richtungsadverbien mit den Suffixen ,-hin“ und „-wärts“ (ebd.: 113f.) (hier: V. 6 und V. 10) kommt auch übersetzerisch voll zum Einsatz. Celans sprachliche und dichterische Kraft wird in der lexikalischen Neukreation ,umschweigen“ (V. 8) augenfällig. [Steiner identifiziert diese Lexeme als Celans „Engführungen“, die „,vor ihm nicht da gewesen sind." ]

\subsection{Die Identität des Originals}

Wie verhält es sich nun mit dem Profil des Originals? Die Analyse hat einen vordergründig bildzentrierten Diskurs in einem vorwiegend beschreibenden Ton gekoppelt mit einer gewissen Zurückhaltung in der Verwendung von Stilmitteln an den Tag gelegt. Werden der Text und sein Autor dadurch identitätslos bzw. „banal“, „konventionell“, „stereotyp“, um mit Steiner zu sprechen? Ein Blick auf die Poetik Supervielles soll bei der Beantwortung dieser Frage helfen.

Supervielles spezifische Auffassung von der Dichtung liegt in zwei poetischen Manifesten - „Eléments d'une poétique“ (1946) 
und „En songeant à un art poétique“ (1951) - sowie in seiner Korrespondenz mit dem Literaturwissenschaftler René Etiemble (1969) vor. Daraus sind folgende drei ästhetische Prinzipien für das vorliegende Anliegen relevant: (1) die simplicité und transparence, (2) die lanterne magique und (3) die technique mouvante:

(1) Simplicité und transparence: Die Gedichte Supervielles stechen durch formale Schlichtheit und Transparenz heraus. Diese sind die zwei Instrumente, mit deren Hilfe der Dichter sein poetisches Universum erkundet und zu deren Tiefen vorzustoßen versucht: „Pour moi ce n'est qu'à force de simplicité et de transparence que je parviens à aborder mes secrets essentiels et à décanter ma poésie profonde“ (Supervielle 1951/1996: 561). Die daraus resultierende, u. U. sogar banal wirkende Einfachheit seines poetischen Diskurses stellt Supervielle in den Dienst der Verständlichkeit, die er sich als oberstes Ziel für seine Dichtung setzt:

Je n'ai guère connu la peur de la banalité qui hante la plupart des écrivains mais plutôt celle de l'incompréhension et de la singularité. N'écrivant pas pour des spécialistes du mystère j’ai toujours souffert quand une personne sensible ne comprenait pas un de mes poèmes. (Supervielle 1951/1996: 561)

Diese bewusste Entscheidung für Transparenz und Klarheit im Gedicht (und die implizite Absage an den Hermetismus, die die moderne Lyrik vielfach charakterisiert) thematisiert Supervielle unmissverständlich in der Theorie der „zwei Pedale“, „la claire“ und „l'obscure“: Das helle Pedal erlaube dem Dichter, „transparence“ herzustellen, das dunkle hingegen reiche bis zur „opacite““. Auf das dunkle habe er nur selten getreten (Supervielle 1951/1996: 561). Die gewollte formale Schlichtheit ist für Supervielle ein Mittel zum Zweck. Sie soll dem Leser den Weg in die Tiefe des Gedichts ebnen, wo Geheimnis und Sinn weilen: ,la surface sera limpide alors que le mystère se réfugiera dans les profondeurs“ (ebd.: 562).

(2) Lanterne magique: Jenem ,centre mystérieux où bat le cœur même de la poésie“" (Supervielle 1951/1996: 561), zu dem dem Leser Zugang erschaffen werden soll, nähert sich der Dichter mittels Bilder: „la plupart du temps je n'avance dans ma pensé qu'à la faveur des images“" (ebd.: 563). Oft baut ein Poem auf einem einzigen Bild auf, die die Funktion einer ,épine dorsale à tout le poème” 
(Supervielle 1946: 31) erfüllt. Diesem kommt die Bedeutung einer „lanterne magique“ (Supervielle 1951/1996: 561) zu. Das poetische Bild als ein Instrument zur Erhellung der Tiefen - und somit als „instrument de connaissance“ (Supervielle 1946: 31) - besitze zwar nicht die Genauigkeit des Begriffs, aber sie verfüge über größere Strahlkraft und dringe tiefer ins Unbewusste als der letztgenannte ein (Supervielle 1951/1996: 563). Zu Supervielles außergewöhnlichen Bildern hat sich auch Rainer Maria Rilke in einem Brief von 28.11.1925 geäußert:

Les images que vous soumettez à vos plus secrètes intentions sont parfois d'une origine tellement éloignée et vous les employez avec une hardiesse d'explorateur : il arrive parfois que mon clavier trop européen sans doute ne contient pas la touche qu'elles voudraient frapper, mais pas un instant je ne doute que cette musique à vide soit pourtant, elle aussi, de la même qualité que celle que, sous vos attouchements précis, mon instrument rend là où il suffit [...]. (Rilke/Supervielle 1950/1952: 49)

(3) technique mouvante: In der bereits erwähnten Linie der Einfachheit steht Supervielles Ablehnung der stilistischen Originalität in der Dichtung (Supervielle 1946: 30). Auffälligkeiten sind nach ihm zugunsten einer „originalité moins consciente“ klassischer Art (Supervielle 1951/1996: 562f.) zu vermeiden. Er schreibe oft „sans penser aux mots“, ja er bemühe sich bewusst um Wortvergessenheit (ebd.: 563), um dem Mysterium der Poesie nah zu kommen. Nicht Glanz, sondern eine gewisse stilistische Verhaltenheit finde er für seine Dichtung angemessen: ,Je n'aime pas en poésie (dans la mienne, du moins) les richesses très apparentes“, vielmehr sollen sie „Sourdes“ und „un peu confuses“ sein, denn: „S'il doit se produire, que le miracle s'avance à pas de loup et se retire de même après avoir fait son coup" (ebd.: 564).

Vielfältig sind die Konsequenzen einer solchen Grundüberzeugung. Tradierte Muster und Vorstellungen von poetischem Register und dichterischer Technik werden von Supervielle hinterfragt und umgestaltet: nicht mittels gekünstelter Rhetorik, sondern mit einem alltäglichen, u. U. sogar umgangssprachlichen Vokabular, das Frieden und Besonnenheit bewirkt, lassen sich die eigenen inneren Ungeheuer zähmen: 
J'ai toujours plus ou moins redouté de m'attaquer aux monstres que je sens en moi. Je préfère les apprivoiser avec les mots de tous les jours, lesquels sont rassurants entre tous. (Ne sont-ils pas ceux-là mêmes qui nous ont tranquillisés lors des grandes peurs infantines?) Je compte sur leur sagesse et leur amitié maintes fois éprouvées pour neutraliser le venin de l'insolite. (Supervielle 1951/1996: 564)

So entsteht in seiner Dichtung eine „rhétorique de l'ordinaire“ (Cook 1997: 42), eine Rhetorik der Alltäglichkeit. Ebenso scheut sein poetischer Diskurs nicht die Nähe zur Prosa, wenn auf diesem Wege Exaktheit, Transparenz, Deutlichkeit besser zu erreichen sind: „Quand j’ai des choses très précises à dire et qui se rapprochent de la prose c'est le verset qui vient, quand je suis dans l'inneffable, le vers" (Supervielle/Étiemble 1969: 34). Auch erhöhen prosaische Einschübe, wenn sie passend betont und rhythmisch gestaltet sind, die Emotionalität eines Moments, denn ,ppar leur grand naturel“" schaffen sie ,un pathétique extraordinaire” (Supervielle 1951/1996: 564).

Supervielles poetische Methode, ,das Gedicht zu sich kommen zu lassen, ohne ihm im Voraus eine Form aufzuzwingen “ (Böschenstein 2001: 268), bedeutet keine grundsätzliche Absage an ästhetische Maßstäbe, denn er überarbeitet seine Gedichte im Nachhinein intensiv. In einem Brief vom 08.12.1939 notiert er, er sei „anarchiste littéraire comme point de départ pour chaque poème, et classique comme point d'arrivée“" (Supervielle/Etiemble 1969: 34). Von der sorgfältigen nachträglichen Korrekturarbeit an den Texten zeugen seine Manuskripte, die ,étonnament travaillés, remaniés, comme fiévreusement, avec cette écriture du désarroi que fut celle de Supervielle, comme agitée d'un trouble incessant qu'il s'efforçait, rature après rature, de maîtriser" (Ray 1995: 5) sind. In den umfangreichen Anmerkungen und Kommentaren Michel Collots, dem Herausgeber von Supervielles CEuvres poètiques complètes, werden Genese, Entwicklung, Varianten eines jeden Poems (Collot in Supervielle 1951/1996: 655-1055; Collot 1993) anhand der Originale sorgfältig nachgezeichnet und festgehalten. Jene ,grâce“ und „apparente facilité“ (Etiemble 1946: 69), die die Verse Supervielles kennzeichnen, sind Resulat einer intensiven Textarbeit und einer konsequenten Suche nach Einfachheit und Natürlichkeit des poe- 
tischen Tones, wobei Supervielle - mit Valéry - gesteht: „Il faut énormément d'art pour être véritablement soi-même et simple“ (Supervielle 1951/1996: 560).

Jules Supervielle - ,eine einprägsame, aber nicht erstrangige literarische Figur" (Steiner/Plessner 2004: 386)? Das Gedicht „Chanson“ - ein Text, der ,wohlgestaltet, aber nicht frei von Banalitäten“ und „Stereotypen“ ist? Vor der Hintergrundfolie der Poetik Supervielles bedeuten diese Einschätzungen ein Missverständnis der Identität dieses Poeten, für den die Einfachheit und die stilistische Verhaltenheit zu dem Wesenskern seiner Dichtungsauffassung gehören.

\subsection{Poetische Interaktion}

Spielt man Texte gegeneinander aus (nach der Lektüre der Übersetzung Celans könne man „kaum zu Supervielle zurück“), so geht dabei zwangsläufig etwas verloren, in diesem Fall das Spezifikum Supervielles. Die neuere translatologische Forschung hält einen Begriff bereit, der einen Ausweg aus dieser binären Optik bietet, nämlich den der poetischen Interaktion. Dieser Terminus behält die Individualität eines jeden Textes im Auge und ermöglicht zugleich deren Zusammendenken.

Der Terminus stammt von Henri Meschonnic (1973) und wurde von Christine Lombez (2009) und Angela Sanmann (2013) vertieft und weiterentwickelt. Er steht für eine produktive Wechselwirkung zwischen Original und Translat. In Abgrenzung vom älteren Postulat der Transparenz im Verhältnis der beiden Texte zueinander (die „treue“ Übersetzung als durchsichtiges Medium, das einen ungetrübten Blick auf das Original erlaubt) definiert Meschonnic seine begriffliche Prägung folgendermaßen: „On lui [sc. la notion de transparence] oppose la traduction comme réénonciation spécifique d'un sujet historique, interaction de deux poétiques, décentrement, le dedans-dehors d'une langue et des textualisations dans cette langue“" (Meschonnic 1973: 307f.; Hervorh. i. O.). In dieser knappen Definition kommen drei für unsere Zwecke klärende Gedanken zum Ausdruck: (1) Eine Übersetzung ist das Ergeb- 
nis der individuellen Auseinandersetzung eines übersetzenden Subjekts, das seinen jeweils spezifischen geschichtlichen Hintergrund in das Translat miteinbringt (,réénonciation spécifique d'un sujet historique ${ }^{\circ}$ ). Die Übersetzung wird somit zur Aussage eines Individuums in der Zeit. (2) Unter dieser Voraussetzung wird sie zum Text (,textualisation“), d. h. zu einem Werk, das kraft seiner Individualität Anspruch auf einen ähnlichen Status wie das Original oder wie andere Werke eines Dichter-Übersetzers erheben darf (vgl. Pennone 2007: 32). (3) Meschonnic suspendiert die tradierten Vorstellungen von Treue und Freiheit, in deren Mittelpunkt das Original stand, und fördert eine dezentrierte Perspektive („décentrement'), in der weder dem Ausgangs- noch dem Zieltext ein Primat zugestanden wird. Vielmehr gestaltet sich die Relation der beiden Entitäten als ein Verhältnis von Text zu Text, d. h. als ein intertextuelles Verhältnis, in dem die Individualität des einen gegenüber dem anderen sich enthüllt. Das Translat wird somit zur dialogischen Interaktion zweier gleichrangiger, individueller wie autonomer Poetiken (,,interaction de deux poétiques" ${ }^{6}$ : derjenigen des Originalautors und derjenigen des Übersetzers. Als, ,intertextueller Austausch poetologischer Konzepte“ (Pennone 2007: 32f.) sei dann auch das künstlerische Eingreifen des Übersetzers im Akt des Übersetzens gerechtfertigt.

Im Lichte dieser Erkenntnis ist Celans Übersetzung des Gedichts „Chanson“" weniger als „Nobilitierung des Originals“ (Sanmann 2013: 73), sondern vielmehr als eigenständiges, wenn auch in vielfältiger Weise auf den Ausgangstext bezogenes Kunstwerk zu verstehen. Auf dieser Grundlage lässt sich seine deutsche Übersetzung als „Manifestation einer spezifischen Lesart des Ausgangstextes" (ebd.: 75) lesen, die sich als Celans individuelle, sprachlich stark pointierte übersetzerische Antwort auf das Original Supervielles konturiert. Die Wechselwirkung zwischen Autor- und Übersetzerpoetik, die offenbar in Celans Translat zu erkennen ist, gilt als ein wesentliches Merkmal dichterischer Übersetzung (vgl. Olschner 1985: 13), das den Ausgangspunkt für die Erforschung des literarischen Eigenwerts der Übersetzung, d. h. ihrer eigenen Literarizität bereitstellt. 
Diese Überlegungen aus heutiger translatologischer Perspektive dürften dem Dichter-Übersetzer Celan selber nicht fremd sein, da sie einen Nerv seiner eigenen Übersetzungspoetik treffen. In der Korrespondenz mit Hans Bender vom 10. Februar 1961 schreibt Celan, Übersetzungen seien „Begegnungen, auch hier bin ich mit meinem Dasein zur Sprache gegangen“" (zit. nach Neuhaus 1984: 54). Diese Aussage weist gleichermaßen auf den Stellenwert der Übersetzungen als integralen Bestandteils eines Werkzusammenhangs sowie auf die vielfältigen Beziehungen der Translate zu Celans eigenen Texten hin. Oft veranschaulicht Celan seine Begegnungen mit den übersetzten Autoren und Gedichten durch die Metapher des Händedrucks, so beispielsweise im Briefwechsel mit René Char: „Merci de me serrer la main - je serre la vôtre“ (Badiou 2015: 150). In diesem bedeutungsvollen, im Zeichen der Annäherung stehenden Motiv offenbart sich Celans ethische Herangehensweise an den zu übersetzenden Text und Autor. Celan sucht durch diese Nähe schaffende Geste das Gespräch mit den fremden Texten und zugleich mit den in und durch diese Texte Sprechenden. Stellt sich dieses Gespräch nicht ein, so scheut Celan nicht davor zurück, Übersetzungsprojekte abzubrechen. Dies geschieht bspw. mit der Übertragung der Poesie von Marianne Moore, von der er bereits Gedichte übersetzt hatte. So schreibt Celan am 16.07.1953 an den Verleger Max Niedermayer:

Gott weiß, dass ich seit Wochen nichts anderes tue, dass ich unablässig versuche, mir einen Zugang zu dieser Poesie zu verschaffen, dass ich Gedicht um Gedicht abtaste, in der Hoffnung, dass das Dichterische dieser Dichtung sich mir endlich erschließen würde - und dass ich zu keinerlei Resultat gelangen kann. [...] Nein, die Poesie von Miss Moore will sich mir wahrhaftig nicht erschließen, der Schlüssel zu ihr bleibt unaufgefunden. (Zit. nach Gellhaus 1999: 20)

Celan sagt die Übersetzung ab und empfiehlt einen anderen möglichen Übersetzer.

Celans Charakterisierung des Übersetzens als „Begegnung“ führt unmittelbar ins Herz seiner eigenen Poetik. Celan spricht an entscheidender Stelle des Meridian, seines bedeutendsten poetologischen Textes von 1960, davon, dass das Gedicht „,im Geheimnis der Begegnung“" stehe (GW III: 198). Innerhalb der Celan-For- 
schung selbst gilt dieses Schlüsselwort als eine ,längst etablierte Kategorie“ (Ivanović 1996: XI) bzw. es wird als eine ,zentrale“ Größe (Schulz 1979) der Celanschen Dichtungstheorie erachtet. Die unterschiedlichen Formen, die die Dialogizität im Werk Celans annimmt sowie seine offenkundige Verwandtschaft mit der Philosophie des Dialogs von Martin Buber (vgl. Kita 2002) und der ethischen Phänomenologie von Emmanuel Levinas (vgl. Olschner 1985: 54f.; Encarnação 2007) wurden ebenfalls freigelegt.

Begegnung ist bei Celan als „Doppelbewegung“" (Olschner 1985: 52) zu verstehen: Sie ist Bewegung auf den fremden (gelesenen bzw. übersetzten) Text und Autor hin und zugleich Rückkehr zur eigenen Dichtung, die dadurch an Profil gewinnt. Diese Dualität der Begegnung spricht exemplarisch aus den Celanschen Versen „Ich bin du, wenn ich ich bin“ (im Gedicht „Lob der Ferne“, GW I, 33), wo die eigene Identität offen und empfänglich für die Identität eines anderen Ich wird und dann bereichert und aufgeklärt zu sich zurückkehrt. Durch Zitate, Anspielungen und intertextuelle Bezüge integriert Celan das in den Texten anderer (übersetzten) Lyrikern Vorgefundene in die eigene Dichtung. Der Leser und Übersetzer Celan als „,wahrnehmendes Du“ vollzieht durch diese Hinund Her-Bewegung zwischen Fremdem und Eigenem letztlich „ein dynamisches Sichbegegnen“" (Olschner 1985: 50).

Celan hat das Gespräch mit Jules Supervielle offensichtlich gesucht - menschlich wie dichterisch. Die persönliche Begegnung der beiden Dichter fand im April 1960 auf Celans Wunsch hin statt. Denise Bertaux, die Tochter Supervielles, berichtet: „Nous avons connu Paul Celan par notre ami Jean Bollack. C'était un homme très attachant. Je me souviens très bien de l'avoir amené chez mon père Jules Supervielle et qu'il s'est agenouillé devant lui. Mon père était à la fin de sa vie“" (zit. nach Harbusch 2005: 111). Von der intensiven Auseinandersetzung Celans mit diesem Autor bezeugt der relativ große Bestand an Büchern von und über Jules Supervielle in Celans Bibliothek, wie aus der detaillierten Auflistung der Titel mit weiteren Erläuterungen in Harbusch (2005: 464-466) hervorgeht. Das Korpus der Supervielle-Übersetzungen Celans - das umfangreichste unter den von ihm übersetzten französischen Poeten 
- umfasst 35 Gedichte. Nur diese Übersetzungen hat Celan selber als eigenständigen Band (1968 in der Insel-Bücherei erschienen) veröffentlicht. Ob dieses Interesse Celans an Supervielle durch eine gemeinsame biographische Gegebenheit - den Tod der Eltern, der tiefe Spuren im Leben und in der Lyrik beider Poeten hinterließ sich restlos erklären lässt, wie von Pennone (2007: 354) vermutet?2 Ob Celan selber Supervielle für einen marginalen, u. U. banalen Poeten hielt?

\section{Fazit und Ausblick}

Der vorliegende Aufsatz hat sich mit George Steiners Verweis auf Paul Celans Übersetzung von Jules Supervielles „Chanson“ im Zusammenhang mit seiner Darstellung transfigurierender, nach oben verratender Übertragungen auseinandergesetzt, wo sie als Paradebeispiel einer Kompensation der Schwächen des Originals durch die Übersetzung aufgeführt wird. Hinterfragt wurden dabei (1) der apodiktische bzw. stark polarisierende Duktus des Kommentars Steiners, der die Vorzüge eines Translats von solchem Rang als die „grausamste Huldigung“ an das eher ,konventionelle“ Original Supervielles versteht sowie (2) seine Auffassung vom auf Reziprozität und Ausgleich abzielenden Verhältnis zwischen Ausgangs- und Zieltext vor der Folie gegenwärtiger translatologischer Vorstellungen von dieser Relation. Dreh- und Angelpunkt der kritischen Auseinandersetzung mit Steiners Position ist die Kategorie der Identität: Übersetzung - und Original - verfügen über eine eigene Literarizität und übermitteln eine spezifische poetische Aussage, die als solche zu erkennen bzw. anzuerkennen sind, ohne gegeneinander ausgespielt werden zu müssen.

$\mathrm{Zu}$ (1): Anders als in der betont wertend-emotionalen Darstellungsweise Steiners, in der die Hochschätzung für den einen

2 Supervielle verliert im Alter von 8 Monaten beide Elternteile an einer Wasservergiftung, Celans Eltern werden 1942 deportiert und im Konzentrationslager ermordet. Diese Erfahrung präge die Beziehung Celans zu Supervielles Dichtung, die sich vielmehr denn ,eine der brüderlichen Nähe und der Identifikation" (Pennone 2007: 354) als eine poetische auffassen ließe. 
Dichter und der Misskredit für den anderen sich gegenüberstehen, wurde eine translatologische Analyse vorgeschlagen, die jeden Text individuell und im Vergleich mittels linguistischer Begrifflichkeit beschreibt mit dem Ziel, ihre jeweilige Identität vorrangig textuell dingfest zu machen. Durch die neutrale (,trockene“) Terminologie der Sprachwissenschaft gewinnt die Deskription an Bodenständigkeit und bewahrt den Kritiker vor rhetorischen Höhenflügen. Zugleich bietet die Mikroanalyse die Möglichkeit, nicht nur auffällige Differenzen, sondern auch feine Nuancenunterschiede (Schleiermachers ,feinste Linie“) zwischen Original und Translat (z. B. Celans durchgehender Gebrauch des nominalen Singulars in der ersten Strophe) aufzuspüren, die letztlich essentiell zur Eigenheit des jeweiligen Textes beitragen. Die analytisch ermittelten Befunde bieten eine exemplarische Grundlage für weiterführende Fragestellungen, beispielsweise: Was „verrät“ die Änderung des Tempus von Futur in Präsens bzw. die Änderung der Bildlichkeit im Schlussteil des Gedichts über Celans Gottesauffassung? In welchem Verhältnis steht diese Stelle mit Celans Gedicht „Zürich, Zum Storchen“ und seinem zur gleichen Zeit wie die Erstellung der „Chanson“Übersetzung geführten Gott-Gespräch mit Nelly Sachs? Eruiert werden kann ebenfalls: Welche Konsequenzen hatte das übersetzerische Gespräch, ja vielleicht sogar „Begegnung“ Celans mit der Dichtung Supervielles für sein eigenes poetisches Schaffen? Welche neuen Einsichten in die poetische Gedankenwelt und Stilistik Supervielles legt der Umweg über die Übersetzungen Celans frei? Solchen Fragen ist weniger in der und mit den Mitteln der Translatologie als vielmehr im Rahmen der literaturwissenschaftlichen Hermeneutik und der Celan- bzw. Supervielle-Forschung nachzugehen.

$\mathrm{Zu}$ (2): Steiner geht in der Bestimmung des Verhältnisses von Ausgangs- und Zieltext von der Grundannahme aus, dass „eine ideale Übersetzung das Original weder vergrößern noch verkleinern dürfte“ (Steiner/Plessner 2004: 387). Konsequenterweise schließt er sein Modell der hermeneutic motion mit der Phase der Wiederherstellung der Parität der zwei Texte ab, die zugleich als ethische Aufgabe zu verstehen ist: „Der Übersetzer, der Exeget, der 
Leser ist seinem Text nur dann treu, gibt ihm verantwortlich Antwort, wenn er um das Gleichgewicht der Kräfte, die Wiederherstellung der intakten Präsenz bemüht ist, die er durch das aneignende Verstehen gestört hat" (Steiner/Plessner 2004: 319). Indem Celans Übersetzung „über Supervielle weit“ (ebd.: 387) hinausgreift, fällt sie aus diesem Rahmen. - Andere Vorstellungen von der Translation sind heutzutage wirksam. Übersetzen wird vielmehr im Lichte der Differenz und Andersheit aufgefasst und diese Kategorien werden nicht nur als inhärent unausweichlich, sondern auch als legitim erachtet. Diese grundlegende Erkenntnis über das Wesen der Translation wird im besonderen Fall der Poeten-Übersetzer noch einmal gesteigert: „Wenn der Dichter übersetzt, bleibt er in seinem Translat ein Dichter“ (Gil 2015: 148) und stellt somit die traditionelle Konzeption von Treue vor neue Horizonte. Celans deutsche Fassung von „Chanson“ zeigt geradezu exemplarisch: Es handelt sich um „die Treue der Übersetzung zu sich selbst - in der Gestalt eines neuen Gedichts“ (Olschner 1985: 12). Das Konzept der „eigenen Identität" des Translats (Gil 2015: 146) erweist sich in diesem Kontext als einen Schlüsselbegriff, der das translatologische Plädoyer für die Anerkennung der literarischen Übersetzung als eigenständige und eigengesetzliche Gattung unterstützen kann. Eine Übersetzung entsteht in der Nachfolge des Originals, stellt aber für die anderssprachigen Leser ,ein neues Original“ (Stroińska 2015: 146) mit eigenem ästhetischem Wert und in diesem Sinne ,a creative literary genre in its own right" (Robinson 2017: 460) dar.

In einer der ersten Rezensionen zu George Steiners After Babel schlussfolgerte George Woodcock: ,we must accept it for the fertility of its suggestions rather than for the finality of its conclusions “ (Woodcock 1975: 328). Die Monographie erwies sich seit ihrer Publikation als ein in vielfacher Hinsicht anregungsmächtiger Grundlagentext der Übersetzungsforschung (vgl. Agnetta/Cercel 2019). Selbst wenn gegenwärtige translatologische Diskurse differente Ausdrucks- und Sichtweisen bevorzugen, geht von Steiners eindringlicher Empfehlung, mit dem (Übersetzungs-)Werk Paul Celans - einem, in dem jene für die Literatur bzw. für die literarische Übersetzung charakteristische „transformative energy” (Ro- 
binson 2017: 460) vortrefflich am Werk ist - sich auch im Rahmen der Übersetzungswissenschaft intensiv zu beschäftigen, einen wertvollen Forschungsimpuls aus.

\section{Quellenverzeichnis}

AgnetTA, Marco / CerCel, Larisa (2019): „George Steiner's After Babel in Contemporary Translation Studies“. In: Church, Communication and Culture 4/3. S. 363-369.

BADIOU, Betrand [Hrsg.] (2015): Paul Celan - René Char: Correspondance 1954-1968 suivie de la Correspondance René Char - Gisèle Celan-Lestrange (1969-1977). Édition établie, présentée et annotée par Betrand Badiou. Paris: Gallimard.

BEALS, Kurt (2014): „Alternatives to Impossibility. Translation as Dialogue in the Works of Paul Celan“. In: Translation Studies 7/3. S. 284-299.

BERMAN, Antoine (1995): Pour une critique des traductions. John Donne. Paris: Gallimard.

BOASE-BEIER, Jean (2014): ,Bringing Home The Holocaust: Paul Celan's Heimkehr in German and English". In: Translation and Literature 23. S. 222-234.

- dies. (2017): „Interpretation and Creativity in the Translation of Paul Celan“. In: CERCEL, Larisa / AgNeTTA, Marco / AMido LozANO, María Teresa [Hrsg.]: Kreativität und Hermeneutik, in der Translation. Tübingen: Gunter Narr. S. 59-76.

BÖSCHENSTEIN, Bernhard (1984): „Anmerkungen zu Celans letzter Übersetzung. John Daive: Weiße Dezimale". In: text + kritik. Zeitschrift für Literatur 53/54, Heft: Paul Celan. S. 84-88.

— ders. (1988): „Supervielle in Celans Fügung“. In: HAMACHER, Werner / Menninghaus, Winfried [Hrsg.]: Paul Celan. Frankfurt: Suhrkamp. S. 222238.

_ _ ders. (2001): ,Jules Supervielle: Le matin du monde“. In: KÖHLER, Hartmut [Hrsg.]: Interpretationen. Französische Gedicbte des 19. und 20. Jabrbunderts. Stuttgart: Reclam. S. 265-274.

CAMPE, Joachim Heinrich [Hrsg.] (1810): Woerterbuch der deutschen Sprache. Vierter Theil. Braunschweig: In der Schulbuchhandlung.

CERCEL, Larisa (2020): „Zum übersetzerischen Hörwerk Paul Celans“. In: AUEROCHS, Bernd / GÜNTHER, Friederike Felicitas / MAY, Markus [Hrsg.]: CelanPerspektiven 2020. Heidelberg: Winter Verlag. S. 115-131.

_ dies. (2021): „Die Performanz der translatorischen Wörtlichkeit. Am Beispiel Paul Celans". In: AgNeTTA, Marco / CerCel, Larisa [Hrsg.]: Text Performances and Cultural Transfer / Textperformances und Kulturtransfer. Bukarest: Zeta Books. S. 165-180. 
CHAMBERLAIN, Lori (2000): ,Gender and the Metaphorics of Translation“. In: VENUTI, Lawrence [Hrsg.]: The Translation Studies Reader. London / New York: Routledge. S. 314-329.

COLloT, Michel (1993): „Écriture et réparation dans l'œuvre de Supervielle“. In: Littérature 90. S. 38-47.

CoOK, Margaret Michèle (1997): ,Jules Supervielle: pour une poétique de la transparence“. In: Études françaises 33/2. S. 35-46.

ENCARNAÇÃO, Gilda (2007): „Fremde Nähe“: Das Dialogische als poetisches und poetologisches Prinzip bei Paul Celan. Würzburg: Königshausen \& Neumann.

ETIEMBLE, René (1946): „L'évolution de la poétique chez Supervielle entre 1922 et 1934“. In: Valeurs. Revue de critique et de littérature 6. S. 51-71. [= Nachgedruckt in: Les temps modernes, août 1950. S. 533-547.]

GellHaus, Axel (1999): „Das Übersetzen und die Unübersetzbarkeit - Notizen zu Paul Celan als Übersetzer“. In: BoDEnHeImer, Alfred / SANDBAnK, Shimon [Hrsg.]: Poetike der Transformation. Paul Celan - Übersetzerer und übersetżt. Tübingen: Max Niemeyer. S. 7-20.

GIL, Alberto (2015): „Translatologisch relevante Beziehungen zwischen Hermeneutik und Kreativität am Beispiel der Übertragungskunst von Rainer Maria Rilke“. In: GIL, Alberto / KIRSTEIN, Robert [Hrsg.]: Wissenstransfer und Translation. Zur Breite und Tiefe des Übersetzungsbegriffs. St. Ingbert: Röhrig Universitätsverlag. S. 143-162.

GREINER, Norbert (2004): Übersetzung und Literatumvissenschaft. Tübingen: Narr.

HallberG, Robert von (2006): „Celan's Universality“. In: Michigan Quarterly Review XLV/2. URL: <http://hdl.handle.net/2027/spo.act2080.0045.214> (13.02.2021).

HarbusCH, Ute (2005): Gegenübersetzungen. Paul Celans Übertragungen französischer Symbolisten. Göttingen: Wallstein Verlag.

HEIDELBERGER-LEONARD, Irène (1985): ,'Translation as the prime art of communication? A note on George Steiner's After Babel". In: DeBusscher, Gilbert / NOPPEN, Jean-Pierre van [Hrsg.]: Communiquer et traduire. Hommages à Jean Dierickx. Bruxelles: Éditions de l'Université de Bruxelles. S. 19-25.

Hermans, Theo (1996): „The Translator's Voice in Translated Narrative“. In: Target $8 / 1$. S. 23-48.

Hubscher-Davidson, Séverine (2018): Translation and Emotion. A Psychological Perspective. London / New York: Routledge.

Ivanović, Christine (1996): Das Gedicht im Geheimnis der Begegnung. Dichtung und Poetik. Celans im Kontext seiner russischen Lektüren. Tübingen: Max Niemeyer.

KHARMAndar, Mohammad Ali (2018): „A Hermeneutic Critique on George Steiner's Hermeneutic Motion in Translation". In: Crossroads. A Journal of English Studies 20/1. S. 83-98. 


\section{Zur Translatologie der von Dichtern übersetzten Gedichte}

KITA, Jadwiga (2002): „Martin Buber und Paul Celan. Zur Poetik des Dialogs“. In: KIRCHER, Hartmut / KŁAŃSKA, Maria / KLEINSCHMidT, Erich [Hrsg.]: Avantgarden in Ost und West. Literatur, Musik, und Bildende Kunst um 1900. Köln / Weimar / Wien: Böhlau. S. 317-336.

KoHLmayer, Rainer (2019): Literaturübersetzen. Ästhetik und Praxis. Berlin u. a.: Peter Lang.

Koskinen, Kaisa (2020): Translation and Affect. Essays on Sticky Affects and Translational Affective Labour. Amsterdam / Philadelphia: John Benjamins.

Lehmann, Jürgen / Ivanović, Christine [Hrsg.] (1997): Stationen. Kontinuität und Entwicklung in Paul Celans Übersetzungswerk. Heidelberg: Winter Universitätsverlag.

LOMBEZ, Christine (2009): L a traduction de la poésie allemande en français dans la première moitié du XIXe siècle: réception et interaction poétique. Tübingen: Niemeyer.

- (2016): La Seconde Profondeur. La traduction poétique et les poètes traducteurs en Europe au XXe siècle. Paris: Les Belles Lettres.

MAY, Markus (2004): „Ein Klaffen, das mich sichtbar macht“. Untersuchungen zu Celans Übersetzungen amerikanischer Lyrik. Heidelberg: Winter.

MesCHONNIC, Henri (1972): „On appelle cela traduire Celan“. In: Cabiers du chemin 14 (15 janvier 1972). S. 115-149. [Wiederabgedruckt in: Ders. (1973): Pour la poétique II. Épistémologie de l'écriture. Poétique de la traduction. Paris: Gallimard. S. 369-405.]

—_ ders. (1973): „Propositions pour une théorie de la traduction“. In: Ders.: Pour la poétique II. Epistémologie de l'écriture. Poétique de la traduction. Paris: Gallimard.

NeuHaus, Volker [Hrsg.] (1984): Briefe an Hans Bender. München: Hanser Verlag.

Nouss, Alexis (2010): Paul Celan. Les lieux d'un déplacement. Paris: Le Bord de l'eau.

Olschner, Leonard M. (1985): Der feste Buchstab. Erläuterungen zu Paul Celans Gedichtübertragungen. Göttingen: Vandenhoeck \& Ruprecht.

PAEPCKE, Fritz (1987): ,Wie verändert Übersetzen ein Gedicht? Charles Baudelaire, La mort des Paurres - Paul Celan, Der Tod der Armen“. In: Celan Jabrbuch 1. S. 183-217.

PENNONE, Florence (2007): Paul Celans Übersetzungspoetik: Entwicklungslinien in seinen Übertragungen franæösischer Lyrik. Tübingen: Max Niemeyer.

POPOVIČ, Anton (1976): „Aspects of Metatext“. In: Canadian Review of Comparative Literature 3/1-3. S. 225-235.

RAY, Lionel (1995): „Deux grandes lyriques“. In: Europe. Revue littéraire mensuelle 792/avril. S. 3-7.

RiLkE, Rainer Maria / SuPERvielle, Jules (1950/1952): „Correspondance“. In: Les lettres. Poésie, philosophie, critique. Revue trimestrielle. Paris: Silvaire. S. 4855.

RoBInson, Douglas (1991): The Translator's Turn, London: The Johns Hopkins University Press. 
__ ders. (2016): Semiotranslating Peirce. Tartu: University of Tartu Press.

- ders. (2017): „What kind of literature is a literary translation?” In: Target 29/3. S. $440-463$.

— ders. [Hrsg.] (2022 i. V.): Cognition and Hermeneutics. Convergences in the Study of Translation (= Yearbook of Translational Hermeneutics / Jabrbuch für Übersetzungshermeneutik, Heft-Nr. 2).

SANMANN, Angela (2013): Poetische Interaktion: französisch-deutsche Lyrikübersetzung bei Friedhelm Kemp, Paul Celan, Ludwig Harig, Volker Braun. Berlin: Walter de Gruyter.

SCHULZ, Georg-Michael (1979): „Individuation und Austauschbarkeit. Zu Paul Celans ,Gespräch im Gebirg“". In: Deutsche Vierteljahrschrift 53. S. 463-477.

SENG, Joachim (2007): ,Mitsprechende Gedankenwelt: Paul Celan als Leser Rudolf Borchardts. München: Stiftung Lyrik Kabinett.

SNell-HornBY, Mary (2006): The Turns of Translation Studies. New Paradigms or Shifting Viewpoints? Amsterdam / Philadelphia: John Benjamins.

STACKELBERG, Jürgen von (2005): „,Mein Ich ging vor mir her'. Rainer Maria Rilke als übersetzerischer ,Egozentriker“". In: Moderne Sprachen 49/1, Themennummer: PÖCKL, Wolfgang [Hrsg.]: Mehrsprachigkeit / Übersetzung. Gedenkschrift für Mario Wandruszka. S. 135-146.

STEINER, George (1978): On Difficulty and Other Essays. Oxford / Melbourne: Oxford University Press.

__ ders. (1989): „North of the Future. On Paul Celan“. In: The New Yorker, 28. August 1989. S. 95.

_ ders. (2009): George Steiner at The New Yorker, edited and with an introduction by Robert Boyers, New York: New Directions.

_ ders. (2011): The Poetry of Thought: From Hellenism to Celan, New York: New Directions.

SteInER, George / Plessner, Monika [Übers.] (2004): Nach Babel. Aspekte der Sprache und des Übersetzens, Frankfurt: Suhrkamp.

STEINER, George / BORNHORN, Nicolaus [Übers.] (2011a): Gedanken dichten, Berlin: Suhrkamp. [= Deutsche Ausgabe von The Poetry of Thought: From Hellenism to Celan, 2011.]

STEINER, George / BORNHORN, Nicolaus (Übers.) (2011b): Im Raum der Stille. Lektüren, Berlin: Suhrkamp.. [= Deutsche Ausgabe von George Steiner at The New Yorker, 2009.]

STOLZE, Radegundis (1992): Hermeneutisches Übersetzen. Linguistische Kategorien des Verstehens und Formulierens beim Übersetzen. Tübingen: Narr.

STROIŃSKA, Dorota (2015): „Sinn und Sinnlickeit. Warum literarisches Übersetzen eine Kunst ist". In: BuschmanN, Albrecht [Hrsg.]: Gutes Übersetzen. Neue Perspektiven für Theorie und Praxis des Literaturübersetzens. Berlin / Boston: Walter de Gruyter. S. 137-152. 


\section{Zur Translatologie der von Dichtern übersetzten Gedichte}

SuPERVIELlE, Jules (1946): „Eléments d'une poétique“. In: Valeurs. Revue de critique et de littérature 5. S. 27-35.

__ ders. (1951/1996): „En songeant à un art poétique“. In: Naissances, poèmes suivis de En songeant à un art poétique. Paris: Gallimard. S. 57-71. [Nachgedruckt in: SuPERVIELLE, Jules (1996): Euvres poétiques complètes. Édition publiée sous la direction de Michel Collot, Bibliothèque de la Pléiade, Paris: Gallimard. S. 559-565).

Supervielde, Jules / ETIEMBLE, René (1969): Correspondance Jules Supervielle-Etiemble. Édition critique. Texte établi, annoté et préfacé par Jeannine Etiemble. Paris: Société d'Édition d'Enseignement Supérieur.

TASHINSKIY, Aleksey (2016): „Das Werk und sein Übersetzer. Translatorische Text-Person-Relationen im Kräftefeld des Originalitätsdispositivs“. In: KELLETAT, Andreas F. / TASHINSKIY, Aleksey / BOGUNA, Julija [Hrsg.]: Übersetzerforschung. Neue Beiträge zur Literatur- und Kulturgeschichte des Übersetzens. Berlin: Frank \& Timme. S. 307-356.

VenUTI, Lawrence (2019): Contra Instrumentalism. A Translation Polemic. Lincoln: University of Nebraska Press.

WOODCOCK, George (1975): „,Review of George Steiner, After Babel: Aspects of L anguage and Translation, Oxford University Press, 1975“. In: Modern Language Quarterly 36/3. S. 324-329.

WOODSWORTH, Judith (2017): Telling the Story of Translation. Writers who Translate. London / New York: Bloomsbury Academic. 